\title{
Squirrel Monkey Lateral Thalamus. I. Somatic Nociresponsive Neurons and Their Relation to Spinothalamic Terminals
}

\author{
A. Vania Apkarian and Ting Shi \\ Department of Neurosurgery, Computational Neuroscience Program, SUNY Health Science Center, Syracuse, New York \\ 13210
}

\begin{abstract}
The incidence and response properties of nociresponsive neurons, their locations relative to spinothalamic terminals, and their relations to cytoarchitectonic borders were studied in the lateral thalamus of the squirrel monkey. Nociceptive neurons were found in ventral posterior inferior nucleus (VPI), in the lateral and medial nuclei (VPL and VPM) of the ventral posterior complex (VP = VPL + VPM), as well as the posterior complex (PO). The overall incidence of nociresponsive cells was $19 \%$ ( 50 of 270 cells). The proportion of nociresponsive neurons within VPI was $50 \%$ (23 of 46$), 38 \%$ in PO (8 of 21), and $10 \%$ in VP (19 of 203). Most nociresponsive cells (90\%) in VP were of wide-dynamic-range type, while within VPI $43 \%$ of nociresponsive cells were nociceptive-specific type. Most of these nociresponsive cells had thermal and mechanical responses, and a small number also responded to cooling. The receptive fields of nociresponsive cells in VPL were in continuity, in both size and body location, with surrounding low-threshold units. The receptive fields of VPI and PO nociresponsive cells were larger than those in VPL. The probability of encountering nociresponsive cells located within $100 \mu \mathrm{m}$ of spinothalamic terminations was high in VPI $(73 \%)$ and low in VPL $(33 \%)$. On the other hand, the probability of encountering non-nociceptive cells located within $100 \mu \mathrm{m}$ of spinothalamic terminals was low in both VPI $(20 \%)$ and VPL $(26 \%)$. The results indicate segregation of nociresponsive cell types across VP, VPI, and PO and suggest that VPI, and perhaps PO, is an important region for discriminative processing and perception of painful stimuli.
\end{abstract}

[Key words: electrophysiology, pain, ventral posterior nuclei, VPL, VPI, PO, nociceptive specific, heating and cooling responses]

Clinical, physiologic, and anatomic evidence implicates various regions of the thalamus in pain perception (Albe-Fessard et al., 1985; Willis, 1985; Besson and Chaouch, 1987; Hodge and Apkarian, 1990). Since the work of Head and Holmes (1911) the role of the somatosensory thalamus in pain perception has

\footnotetext{
Received Dec. 16, 1993; revised May 3, 1994; accepted May 10, 1994.

We thank Dr. K. D. Kniffki and Dr. C. J. Hodge for participating in some of these experiments, and Dr. J. Dostrovsky for comments on a earlier version of the manuscript. The technical help of Dr. A. Stea and R. T. Stevens is appreciated. This research was funded by the Department of Neurosurgery and by a grant from the Perkins foundation.

Correspondence should be addressed to A. Vania Apkarian, SUNY Health Science Center at Syracuse, Neurosurgery Research Laboratory, 3118 WSK Hall, 766 Irving Avenue, Syracuse, NY 13210.

Copyright (C) 1994 Society for Neuroscience $0270-6474 / 94 / 146779-17 \$ 05.00 / 0$
}

been functionally subdivided into a medial and a lateral portion. Currently, it is accepted that medial thalamic regions with nociceptive responses are mainly involved in motivational and affective properties of pain, while the lateral thalamic nuclei with nociceptive responses are presumed to process and transmit discriminative aspects of noxious stimuli (see, e.g., Melzack and Casey, 1968; Price and Dubner, 1977; Craig and Burton, 1981; Kniffki and Mizumura, 1983). For a number of years we have been charting the anatomy of nociceptive information transfer by the spinothalamic pathway through the thalamus to the cortex (Gingold et al., 1991; Shi et al., 1993; Stevens et al., 1993) in the squirrel monkcy. These studies imply that different subdivisions of the lateral thalamus receive and transmit specific types of nociceptive information to specific subdivisions of the somatosensory cortex. The latter hypothesis can be tested directly by studying the nociceptive response properties of the neurons within these thalamic areas.

A number of investigators have described the nociresponsive properties of lateral thalamic neurons in multiple species. These studies show important species differences in the location and topography of these neurons in the lateral thalamus. Nociresponsive cells have been described in the core of the ventral posterior complex (VP) in both rat and monkey and the large majority of these are wide-dynamic-range type (WDR; Guilbaud et al., 1980; Kenshalo et al., 1980; Chung et al., 1986; Casey and Morrow, 1987). On the other hand, nociresponsive cells in the cat seem to be confined to the ventral and dorsal border regions of this region (VP is designated as ventrobasal complex in cat and raccoon; Honda et al., 1983; Kniffki and Mizumuna, 1983; Kniffki and Craig, 1985; Yokota et al., 1988). Here, too, the majority of cells were described as WDR type although the relative locations of WDR type and nociceptivespecific (NS) type neurons were reported either as segregated (Yokota et al., 1988) or intermixed (Kniffki and Mizumuna, 1983; Kniffki and Craig, 1985). Recently, Simone et al. (1993) reported on the properties of nociresponsive cells in the raccoon lateral thalamus. They describe mostly WDR and some NS type cells within VP, dorsal and ventral borders of VP, and the medial division of the posterior nucleus (POm). They were unable to isolate units in the ventral posterior inferior nucleus (VPI) of the raccoon. Casey and colleagues have studied nociresponsive cells in the lateral thalamus of the squirrel monkey (Casey, 1966; Casey and Morrow, 1983, 1987; Morrow and Casey, 1992). They describe only WDR type cells located mainly in the core of VP. However, these studies were done in unanesthetized behaving monkeys, where frequent intense noxious stimuli could not be used. Therefore, it is unclear whether their results were 

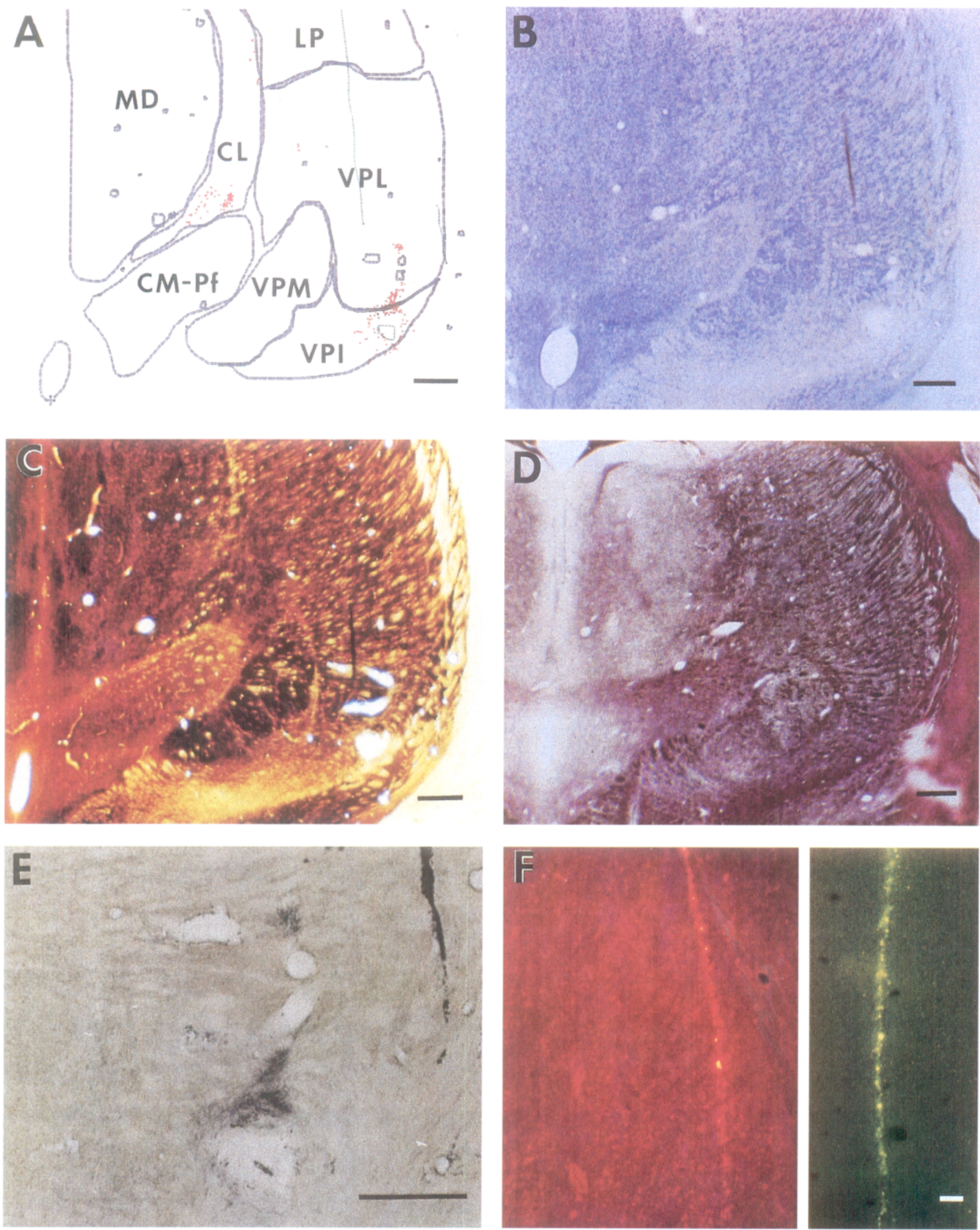

Figure 1. Electrode tracks, spinothalamic terminations, and cytoarchitecture of VPL and VPI. $A$, Computer reconstruction of a thalamic section with cervical enlargement spinothalamic terminals ( red dots) and an electrode track with a lesion (green) marking the location of a nociceptive 
due to the testing paradigm or are a true reflection of the lack of NS type nociceptive units in the lateral thalamus of the awake monkey.

The squirrel monkey lateral thalamus has a large well-differentiated VPI. This facilitates studying the properties of neurons in VPI and comparing them to neurons located in the ventral posterior lateral (VPL) or medial (VPM) nuclei (see below, and Gingold et al., 1991; Stevens et al., 1993). In comparison, the VPI in Old World monkeys seems relatively smaller and the borders between VPI and VP are also less clear (Jones, 1985). In contrast to the ventral periphery of the cat VP (an area equivalent to monkey VPI; see Kniffki and Craig, 1985; Vahle-Hinz et al., 1987; Vahle-Hinz and Kniffki, in press), the response properties of VPI cells in the monkey have not been systematically studied with noxious stimulation. In this study, we examine the nociceptive properties of neurons in VPL, VPI, and the posterior complex (PO) in the squirrel monkey. Within this region, every isolated unit was tested to determine adequate somatic mechanical stimuli and classified as non-nociresponsive or nociresponsive. This approach enabled us determining the frequency of encountering nociresponsive cells relative to various lateral thalamic cytoarchitectonic boundaries. Nociresponsive cells were also studied to determine their thermal response properties. Moreover, in most animals spinothalamic (STT) terminals were labeled with wheat germ agglutinin-conjugated horseradish peroxidase (WGA-HRP). The proximity between recording sites and STT terminals was analyzed to determine the relative contribution of the STT to the nociresponsive and non-nociresponsive neurons in the lateral thalamus.

\section{Materials and Methods}

Anesthesia and preparation. Single-unit electrophysiologic experiments were done in seven adult female squirrel monkeys (weighing $0.45-0.74$ $\mathrm{kg}$ ) anesthetized with $\alpha$-chloralose/Nembutal. Initially, single-unit physiologic studies were done in intact animals (two animals). In later studies the animals received a prior injection of WGA-HRP into the cervical enlargement (five animals). After a $5 \mathrm{~d}$ survival timc, they were reanesthetized and single-unit physiologic recordings were done in the lateral thalamus.

The housing, care, and surgical procedures followed the institutional guidelines established by the Committee for the Humane Use of Animals. The initial surgery for WGA-HRP injections was done using strict sterile procedures in a sterile animal operating room. Individually housed monkeys were pretreated with dexamethasone $(0.25 \mathrm{mg} / \mathrm{kg}$, i.m., twice daily) to prevent the development of edema and an antibiotic (Rocephin, $75 \mathrm{mg} / \mathrm{kg}$, i.m.). Each animal was anesthetized with ketamine $(30 \mathrm{mg} /$ $\mathrm{kg}$, i.m.) and with Nembutal (10 $\mathrm{mg} / \mathrm{kg}$, i.v.); supplemental doses of ketamine $(10 \mathrm{mg} / \mathrm{kg} / \mathrm{hr}, \mathrm{i} . \mathrm{m}$.) and Nembutal drip $(0-10 \mathrm{mg} / \mathrm{kg} / \mathrm{hr}, \mathrm{i} . \mathrm{v}$.) were given to maintain a surgical level of anesthesia. Intravenous fluid replacement with $5 \%$ dextrose lactated Ringer's solution was given during surgery. The animals were intubated to ensure a patent airway. Body temperature, expired $\mathrm{CO}_{2}$, oxygen saturation (using a pulse oximeter), heart rate, and blood pressure (using an automated cuff) were monitored noninvasively and maintained within physiologic limits. Animals received a single dose of antibiotics during surgery (Rocephin, $75 \mathrm{mg} / \mathrm{kg}$, i.m.). Following surgery the animals were administered antibiotics for $3 \mathrm{~d}$ and checked daily to ensure normal recovery. Animals that exhibited symptoms of pain during recovery period were administered analgesics (Torbugesic, $0.05 \mathrm{mg} / \mathrm{kg}$, i.m.).

Following anesthesia induction, the monkey was placed in a stereo- tactic frame and a laminectomy done to expose the cervical enlargement of the spinal cord. Two percent WGA-HRP (Sigma) was pressure injected in the spinal cord using a $20-40-\mu \mathrm{m}$-tip glass micropipette connected to a picospritzer. Injections were made $1 \mathrm{~mm}$ from midline at two depths ( $0.5-1.0$ and $1.5-2.0 \mathrm{~mm}, 0.15 \mu \mathrm{l} / \mathrm{site})$, on the right side. A total of 10 injections were made in segments C5-T1. Following the injections the wound was infiltrated with antibiotics and closed.

After a $5 \mathrm{~d}$ survival, the animal was reanesthetized initially with a small dose of ketamine $(30 \mathrm{mg} / \mathrm{kg}, \mathrm{i} . \mathrm{m}$.), followed by a full dose of $\alpha$-chloralose $(70 \mathrm{mg} / \mathrm{kg}$, i.v.) and sustained with a continuous drip of Nembutal $(0-10 \mathrm{mg} / \mathrm{kg} / \mathrm{hr}$, i.v.). Supplemental doses of $\alpha$-chloralose (35 $\mathrm{mg} / \mathrm{kg}$, i.v.) were given every $12-14 \mathrm{hr}$. Tracheal, arterial, and venous cannulas were placed, the animal put in a stereotactic head-holder, and a cranial clamp attached by screws and dental cement, after which the ear bars and mouth piece were removed. Blood pressure, heart rate, expiratory $\mathrm{CO}_{2}$, oxygen saturation, and core body temperature were monitored and maintained within physiologic range. A large bilateral craniotomy was done to provide access to the thalamus.

The recordings in one animal were done after paralyses with gallamine triethiodide (20-30 mg, supplemented every 1-2 hr), and artificial respiration. However, in unparalyzed animals, it was noticed that the anesthetic state of the animal was very stable and rarely showed withdrawal reflexes when the skin was pinched, implying that paralysis was not necessary. Thus, in the following animals, unit recordings were done in unparalyzed animals breathing naturally. This approach enabled us a more accurate control over the anesthetic state of the animals; whenever the animals exhibited motor responses to the noxious stimuli the Nembutal drip rate was increased by $10-20 \%$ for $2-5 \mathrm{~min}$.

Recordings were done with parylene-coated tungsten electrodes (impedance at $1000 \mathrm{~Hz}: 1.5 \mathrm{M \Omega}$; Micro Probe Inc., Clarksburg, MD), which were further coated with nontransportable fluorescent beads in wax (Fig. $1 F$ ). One side of the brain was used to explore the thalamus and determine the location and borders of VPL, using innocuous skin stimulation. Then, in the opposite thalamus, lateral thalamic units were studied systematically. The forelimb portion of VPL was the primary recording target because the cervical WGA-HRP injections primarily label terminals in this region of the lateral thalamus. Ongoing and response activity was stored on tape and, with a window discriminator, converted to uniform pulses that were analyzed on line and off line using an HP1000/A900 computer (see Martin et al., 1990; Smith et al., 1991).

Mapping and classification of response properties. Adequate somatic mechanical response properties and receptive field locations were determined for every isolated unit in every track. Units were characterized only if ncuronal activity was isolated and detcrmincd to originate from the soma-dendritic region, as judged from the shape and duration of the action potentials. Mechanical stimuli included touch (movement of a smooth probe across the skin), brush (bristles, $5 \mathrm{gm}$ ), pressure (large forceps or weights, $50-200 \mathrm{gm}$ on a 1-cm-diameter area), pinch (serrated clamp or forceps, over $1000 \mathrm{gm}$ on a 1-3-mm-diameter area), tap, and deep tissue squeeze. The pinch stimulus was painful to the investigators. Many units that did not exhibit nociceptive responses by mechanical testing were not studied further. Units with nociceptive mechanical responses were studied by thermal stimuli as well. Nonquantitative exploratory responses to thermal stimuli were studied by applying stainless steel probes, heated or cooled to various temperatures (about $-20^{\circ} \mathrm{C}$, $0^{\circ} \mathrm{C}, 20^{\circ} \mathrm{C}$, and $55^{\circ} \mathrm{C}$ ), to the receptive field of the unit. Thermal stimuli were also applied through a Peltier thermode (contact area, $13.2 \mathrm{~cm}^{2}$ ), the temperature of which could be reproducibly controlled from $0^{\circ} \mathrm{C}$ to $54^{\circ} \mathrm{C}$. An adaptive temperature of $25^{\circ} \mathrm{C}$ or $35^{\circ} \mathrm{C}$ was used before heating or cooling the skin. Isolated units were also tested for Pacinian type responses by applying $125 \mathrm{~Hz}$ and $256 \mathrm{~Hz}$ tuning forks onto the isolated units' receptive fields.

Units were classified according to their responses: low-threshold (LT) units were maximally driven by innocuous cutaneous stimuli (touch and/or brush) and/or innocuous pressure; nociceptive specific (NS) units responded only to noxious stimuli (pinch and/or noxious heat); WDR units responded to both innocuous and noxious stimuli, with an obvious

specific type unit in VPI. $B$, Photomicrograph of the same section (as in $A$ ), stained with cresyl violet. $C$, A cytochrome oxidase-stained thalamic section just adjacent to the section in $B . D$. A myelin-stained thalamic section corresponding to the sections shown above. $E$, Photomicrograph of the unstained VPL and VPI region of the section shown in $B$. The lesion was located in VPI and was surrounded with spinothalamic terminals (black). Note the corresponding blood vessels and the lesion in $A-C$ and $E$. F, Recording tracks labeled by red (left) or green (right) microspheres mixed with wax. For abbreviations in this and the following figures, see Appendix. Scale bars: $A-E, 0.5 \mathrm{~mm} ; F, 1.0 \mathrm{~mm}$. 
increase in firing rate to higher intensity stimuli; units with responses to manipulation of a joint, muscle, or subcutaneous tissue were classified as deep. Responses to thermal stimuli further subdivided these classifications into innocuous and noxious heating and cooling responsive units. Response types were determined both subjectively and by measuring the mean change in the rate of the unit response. A given unit was classified as responsive to a specific stimulus only if the responses could be reproduced at least three times and if its mean rate changed significantly by $t$ test at $p<0.05$ level and by at least $30 \%$ (measured over $10 \mathrm{sec}$ period, immediately before and during a stimulus).

Localization of recording sites and their relation to spinothalamic terminals. Recording tungsten electrodes were coated with nontransportable fluorescent dyes of different colors. Figure $1 F$ shows recording tracks labeled by red and yellow microspheres. This color coding of electrodes facilitated localization of the recording sites. The end of the recording tracks and some unit recording sites were also marked by electrolytic lesions. At the end of the experiment, the animal was overdosed with Nembutal $(200 \mathrm{mg} / \mathrm{kg}$, i.v. ) and perfused transcardially. The tissue was then processed for WGA-HRP label and electrode tracks were reconstructed from the fluorescent tracks and the electrolytic lesions.

The animals were perfused first with heparinized normal saline, then with $2.5 \%$ paraformaldehyde, followed by a $10 \%$ glycerol wash. The tissue was stored in a cryoprotective solution for 1 week (Rosene et al., 1986). Serial frozen sections $(50 \mu \mathrm{m})$ were cut from the thalamus (coronal) and the spinal cord (transverse). Thalamic and spinal sections were reacted with tetramethyl benzidine to reveal WGA-HRP labeling (Mesulam, 1978). Alternate thalamic sections were stained for cytochrome oxidase (Wong-Riley, 1979), acetylcholinesterase (Togo et al., 1986), or myelin (Schmued, 1990) to aid the determination of thalamic nuclear boundaries. Spinal cord sections were examined to determine adequacy of injections.

The WGA-HRP labeled spinothalamic terminals were plotted from the reacted sections using a computerized system. These sections were then stained for cresyl violet, and thalamic nuclear boundaries superimposed on the digitized plots, using blood vessels and section boundaries as landmarks. Nuclear boundaries were determined using the same procedures as presented before, by comparing the cresyl violet tissue with the cytochrome oxidase-/and myelin-stained neighboring sections and comparing the terminal label and stained tissue to our earlier anatomic results (Gingold et al., 1991; Stevens et al., 1993). Electrode tracks and electrolytic lesion sites were also superimposed on these plots. On the basis of the electrolytic lesions, the recording sites along the tracks were determined by interpolation. The final resultant plots then had the spinothalamic terminals and the locations of the recording sites on the same sections. Computer software developed earlier (Gingold et al., 1991) was used to determine the recording sites located within 100 $\mu \mathrm{m}$ of spinothalamic terminals. The distance of $100 \mu \mathrm{m}$ was used here based on the work of Ralston et al. (1985), which determined that most STT terminals contact thalamic dendritic profiles within $100 \mu \mathrm{m}$ of their soma. Cell recordings at these sites were called "overlapping cells" and were assumed to putatively receive spinothalamic terminals (for more details, see Gingold et al., 1991).

\section{Results}

\section{Spinal cord injections}

The spinal cord injections of WGA-HRP were done using finetipped glass pipettes that minimized spinal cord damage and produced no differences in the numbers of nociceptive neurons observed in animals without WGA-HRP injections. The injections completely covered the cervical enlargement, occasionally extended to more rostral and caudal segments, and also spilled over into the deep laminae of the spinal cord ipsilateral to the thalamic recordings.

\section{Lateral thalamic nuclear boundaries and nomenclature}

The squirrel monkey lateral thalamus has a prominent VPI, located ventral to VP. The dorsoventral boundaries of VPI can be clearly discerned using sections stained for cresyl violet, cytochrome oxidase, and myelin (Fig. $1 B-D$ ). In cresyl violetstained tissue, VPI cells appear smaller than the dorsal VPL and
VPM cells and the region is lightly stained. With cytochrome oxidase staining, VPI is again lightly stained and the borders well differentiated. The overall myelin pattern in VPI is similar to VPL. In frontal scctions, both regions have fascicles of myelin traveling in the mediolateral direction. In VPI, these fascicles are of shorter length than those in VPL. More anteriorly, there is a density of undifferentiated myelin labeling that delineates the dorsal border of VPI from VPL. A similar but denser undifferentiated myelin region borders VPI ventrally to form the external medullary lamina. Posteriorly, VPI is continuous with PO. The exact boundary between them is not obvious in cresyl violet-stained sections. We arbitrarily assign the posterior limit of VPI at the coronal level where the medial geniculate nucleus begins and VPM disappears. This is the level where the cell morphology begins to change from the typical VPI cells to PO cells. Both the density and size of the cells in VPI are more uniform and smaller, while PO has large neurons mixed with smaller cells. The body of VPI is clearly differentiable from the body of PO by cytochrome oxidase stain, which shows a more complex staining in PO and a uniform pale staining in VPI. Myelin staining also distinguishes PO from VPI and VPL since $\mathrm{PO}$ appears more uniform and is not fasciculated.

In the present study, we were primarily interested in comparing nociceptive response properties of cells in VPL to those located in VPI. Therefore, the recording tracks were directed to VPL, which would ventrally traverse VPI. However, a small number of these tracks were posterior enough such that ventrally the electrodes were located in anterior PO rather than VPI. Our recordings within $\mathrm{PO}$, therefore, were limited to this anterior most region, which we designate $\mathrm{PO}$.

Only three to five recording tracks were done in each animal in order to minimize damage and distortion of the tissue. Lack of tissue distortion is evident in Figure $1 B$, where the electrode track can be seen in the cresyl violet-stained section. The electrolytic lesion, placed at a site where a nociresponsive unit was found, is surrounded by WGA-HRP-labeled spinothalamic terminals (Fig. $1 A, E$ ). This lesion can be seen in the neighboring cytochrome oxidase-stained section (Fig. 1C).

\section{Response propertics}

A total of 270 neurons were studied in VPL, VPM, VPI, and PO. The distributions of all the cells within these nuclei and their response properties to mechanical stimulation are summarized in Table 1 . Of these cells, 220 had purely non-nociceptive responses and 50 had nociceptive responses. Forty-four of the non-nociresponsive cells responded to deep tissue stimulation, four of which also had skin inputs. Most of the cells with deep inputs were located in anterior and dorsal VPL and anterior VPI. The dorsal VPL where most cells have deep inputs is the region called ventral posterior superior by Kaas and Pons (1988). Of the nociresponsive cells, 36 were WDR type and 14 NS. One NS cell, located in VPI, responded to deep noxious squeeze. Most of the NS units had ongoing activity and could only be driven by noxious stimuli. A smaller number had no, or very low, ongoing activities and were discovered only by intense mechanical or thermal stimuli. Since these stimuli cause obvious skin damage, they were applied sparingly. As a result, the response properties, and the extents of the receptive fields of the NS units could not be completely specified. Moreover, the incidence of the NS type cells is probably underestimated. The majority of the isolated cells were also tested for Pacinian 
Table 1. Distribution of cells in the lateral thalamus and their response properties as determined with mechanical and/or thermal stimulation

\begin{tabular}{|c|c|c|c|c|c|c|c|c|}
\hline & \multicolumn{2}{|l|}{$\mathrm{VP}$} & \multicolumn{2}{|c|}{ VPI } & \multicolumn{2}{|c|}{ PO } & \multicolumn{2}{|c|}{ Total } \\
\hline & $\#$ & $\%$ & \# & $\%$ & $\overline{\#}$ & $\%$ & $\overline{\#}$ & $\%$ \\
\hline Non-noc. & 184 & 90.6 & 23 & 50 & 13 & 61.9 & 220 & 81.5 \\
\hline WDR & 17 & 04 & 13 & 50 & 6 & 381 & 36 & 185 \\
\hline NS & 2 & 9.4 & 10 & 50 & 2 & 38.1 & 14 & 18.5 \\
\hline Total & 203 & 100 & 46 & 100 & 21 & 100 & 270 & 100 \\
\hline
\end{tabular}

\#, number of cells in a category; \%, percentage of cells within a category. The percentage of cells is calculated for all nociceptive cells, that is, \%(WDR + NS)/(WDR + NS + Non-noc.). Non-noc., cells that do not show responses to noxious stimulation; WDR, wide-dynamic-range type nociresponsive cells; NS, nociceptive-specific type nociresponsive cells.

type inputs. None of the units tested (in VPL, VPI, or PO) showed a one-to-one response to these stimuli.

The incidence of the nociresponsive cells recorded in VP was different from those in VPI and PO. Seventeen of 189 cells $(9.0 \%)$ recorded in VPL and 2 of 14 cells $(14.3 \%)$ in VPM responded to noxious mechanical stimuli. The rest responded only to innocuous mechanical stimuli. Less than $10 \%$ of the studied units in the two nuclei were nociresponsive. In contrast, 23 of 46 units $(50 \%)$ recorded in VPI and 8 of 21 cells $(38.1 \%)$ in $\mathrm{PO}$ were nociresponsive. Statistical analysis of the response types, for units in VP versus VPI, with innocuous versus noxious responses shows a significant difference between the two regions $\left(\chi^{2}\right.$ test, $\left.p<0.001\right)$. Similar statistical analysis between VP units and PO units shows significant difference in incidence of nociresponsive cells $\left(\chi^{2}\right.$ test, $\left.p<0.001\right)$.

The nociresponsive cells in VP were different from those in VPI. Of the 19 nociresponsive cells found in VP, only two were NS type; one NS type unit was in VPL and the other was located in the lamina between VPL and VPM (see Fig. 2). In contrast, 10 of the 23 nociresponsive cells in VPI were NS type. Most NS type cells were located well within the boundaries of VPI, while many of the WDR units were on the dorsal border of VPI, near the ventral border of VPL. The ratio of NS versus WDR cells located in VP as compared to VPI was significantly different (Fisher's Exact, $p<0.05$ ). Two of the eight nociresponsive cells in PO were NS type.

The activity of a LT neuron located at the ventral border of VPL is shown in Figure $3 A$. The neuron responded maximally to brushing within its receptive field while pressure and pinch resulted in smaller increases in its ongoing activity. A WDR type neuron located in VPI is shown in Figure $3 B$. Increasing the intensity of the mechanical stimulation results in higher response activity. This is the same cell whose location was marked by a lesion shown in Figure $1, A-C$ and $E$. The responses of an NS type cell located in PO is shown in Figure $3 C$. Brushing or pressure applied within the receptive field had no effect on the ongoing activity while noxious pinching increased its activity.

\section{Incidence of nociresponsive cells per track}

The physiological recordings in each of six animals was done within a $24 \mathrm{hr}$ period while in one animal the recording lasted $36 \mathrm{hr}$. The incidence of nociresponsive units encountered seemed independent of the time from the beginning of the experiment. Figure 4 shows the incidence of recording nociresponsive units in each track in all experiments. The tracks are numbered consecutively in each experiment, such that larger numbers correspond to longer time from the start of the experiment. Com- paring the incidence of nociresponsive cells between the tracks shows no time-dependent trends, implying the lack of sensitization.

\section{Thermal responses}

Twenty-three of 40 cells with nociceptive responses to mechanical stimulation also responded to noxious heating of their receptive fields, and 9 of 23 cells tested responded to noxious cooling. Nine of these responded to noxious heating and cooling (seven were WDR type and two were NS type). All except one of these neurons with noxious thermal responses also responded to mechanical noxious stimuli as well. These neurons were found in VPL, VPI, and PO. The neuron without noxious mechanical response was in VPI. Three of these neurons classified as WDR type were located at the border between VPL and VPI and had phasic and sustained responses to innocuous cooling. These are the first cooling responsive units characterized in the monkey thalamus with body receptive fields. Mechanical and thermal responses of a NS type unit located in VPI are shown in Figure 5. Only noxious mechanical stimulation increases the unit activity. The unit had a thermal threshold above $52^{\circ} \mathrm{C}$. Note that the unit's activity increased during the cooling phase following noxious heating, hinting that the unit may respond to innocuous cooling. This unit, in fact, repeatedly responded to cooling of

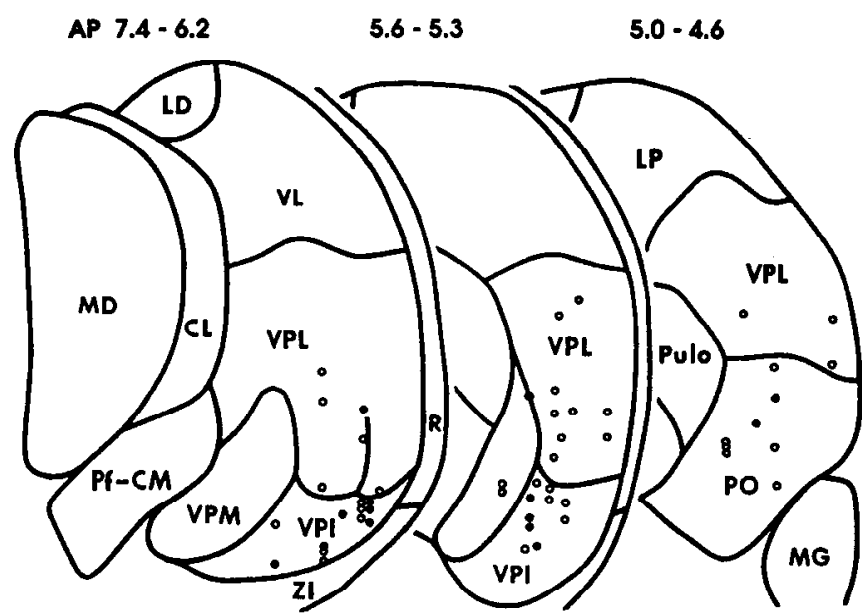

Figure 2. The locations of the nociresponsive cells are summed in three coronal sections of the thalamus. The nociceptive-specific type neurons are shown as solid circles. The wide-dynamic-range type neurons are shown as open circles. The anteroposterior range included within each section is indicated below the corresponding drawing. 

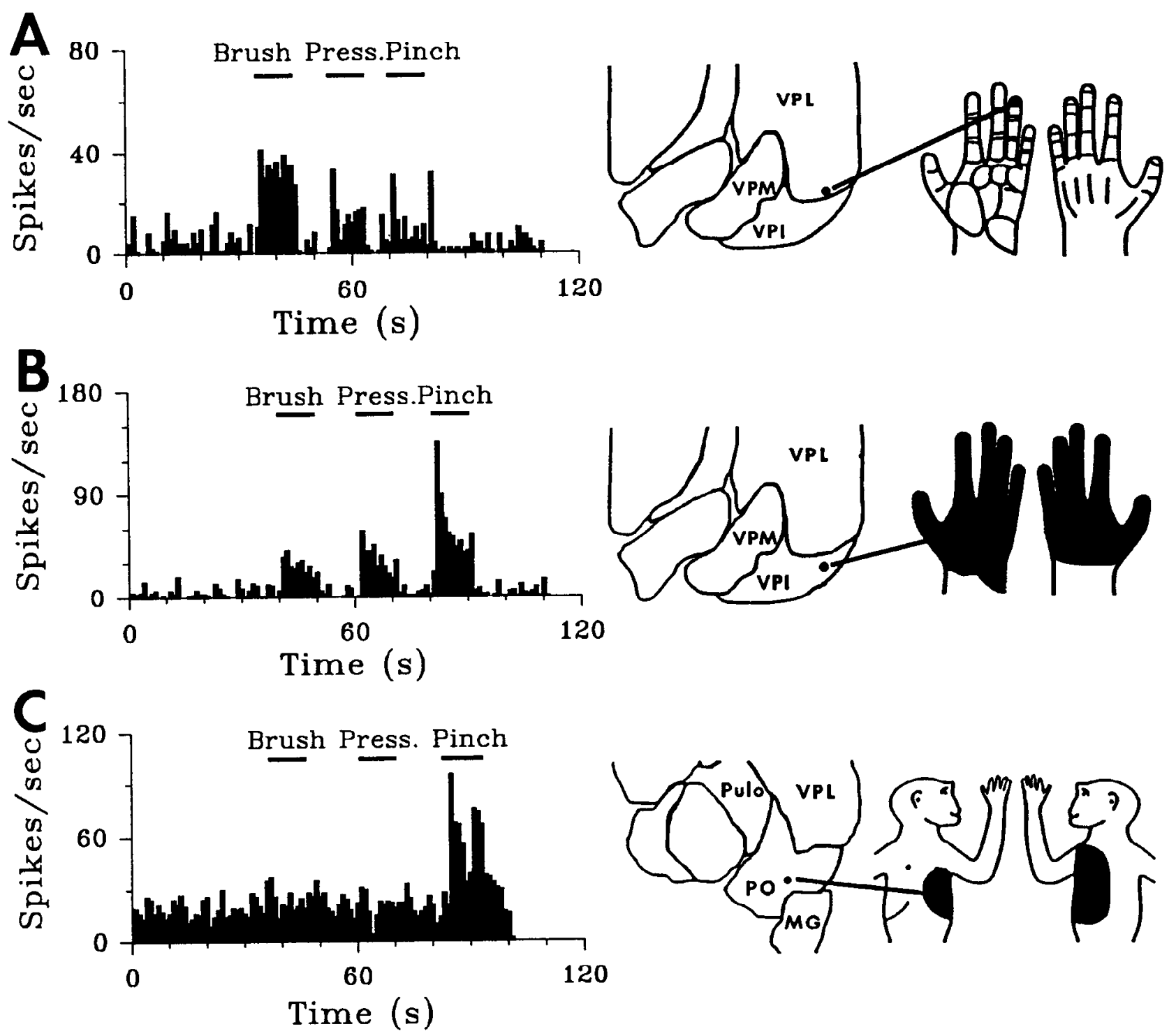

Figure 3. Peristimulus time histograms during mechanical stimulation, thalamic recording locations, and receptive fields are shown for three neurons. $A$, A neuron, classified as non-nociceptive, had a receptive field at the tip of digit 4 , and was located just above the ventral border of VPL. This unit had a brisk response to brushing of the skin and a reduced response to pressure and pinch. $B$, A neuron, classified as wide-dynamicrange type, had a receptive field that covered the whole contralateral hand, and was located in VPI. The unit activity increased with increasing the intensity of the stimulation from brushing to innocuous pressure to noxious pinching. $C$, A neuron, classified as nociceptive-specific type, had a receptive field that covered contralateral chest and back, and was located in PO. The unit activity increased only during noxious pinching.

the skin (from $25^{\circ} \mathrm{C}$ ) by $5^{\circ} \mathrm{C}$ or $10^{\circ} \mathrm{C}$. The responses of a unit in VPI are shown in Figure 6. This unit was classified as WDR since it responded to brushing, pressure, and pinching, with a higher discharge rate to pinch and also responded with a higher rate to a noxious thermal stimulus (around $55^{\circ} \mathrm{C}$ probe). Cooling within the receptive field, from $25^{\circ} \mathrm{C}$ to $15^{\circ} \mathrm{C}$ to $5^{\circ} \mathrm{C}$, also increased its activity.

The thermal nociceptive neurons were evenly distributed between VPL and VPM, and VPI. Within VP, 17 cells were tested for thermal responses, of which 6 were heat responsive and three were responsive both to heating and cooling (in VPM, one of three tested was heat responsive). All nine thermoresponsive units were WDR type. In VPI, 18 cells were tested for thermal responses, of which four WDR and two NS type units were heat responsive, and three WDR and two NS type units were responsive to heating and cooling. In PO, five units were tested, of which three WDR units were heat responsive, and one WDR type unit was responsive to heating and cooling.

\section{Mechanical responses}

The mean spontaneous activity of LT neurons (all but one located in VPL) was $11.4 \pm 9.0$ spikes/sec (mean \pm SD, $n=12$ ), for WDR neurons (in both VPL and VPI) was $10.0 \pm 9.0(n=$ 18 ), and for NS neurons (four in VPL and one in PO) was 9.2 $\pm 6.5(n=5)$. The individual and population stimulus response curves for mechanical innocuous and noxious stimulations are shown in Figure 7. For LT type neurons (11 in VPL and 1 in VPI, Fig. $7 A$ ) the ongoing activity increased during brushing in all cases. With sustained pressure, the ongoing activity of these LT type neurons either increased (presumed slowly adapting type units) or remained unchanged (presumed rapidly adapting type units). During noxious pinching, the increase in unit ac- 


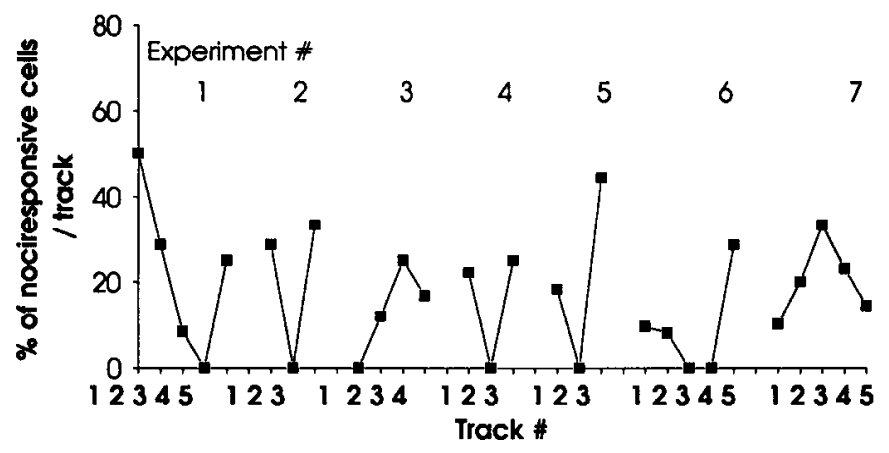

Figure 4. The incidence of nociresponsive cells per track is shown for all tracks in all animals. The experiment number indicates the seven animals used. For each animal, the track numbers are consecutive, larger numbers corresponding to later times in the experiments. The number of neurons recorded per track was $10.8 \pm 6.9$ (mean \pm SD).

tivity was never above the response to brush or pressure. The mean population response increased during brushing $(275 \%$ of ongoing activity, $31.4 \pm 14.9$ spikes/sec) and there was no further increase with pressure or pinch, consistent with the definition of LT type neurons. The WDR type cells in both nuclei have similar response properties. These cells' ongoing activities usually increased during brushing (one exception), either increased or remained unchanged during pressure, and further increased during noxious pinching to a level above the responses to either brushing or pressure. As a result, the population responses for WDR cells in VPL $(n=9$; Fig. $7 B)$ and VPI $(n=$ 9; Fig. 7C) show increased activity during brush (232\% of ongoing activity, $23.20 \pm 15.0$ spikes/sec), no further change during pressure, and increased activity during pinch (531\% of ongoing activity, $53.1 \pm 28.3$ spikes/sec). For the NS units (four in VPI and one in PO; Fig. 7D), individual and population responses showed no, or very small, changes in activity during brushing or pressure, but increased activity during pinching ( $336 \%$ of ongoing activity, $30.9 \pm 23.9$ spikes/sec).

Since the nociresponsive cells were originally identified by mechanical stimuli, and only some of them were responsive to thermal stimuli, it is likely that we missed another population of nociresponsive units, namely, those that are nonresponsive to mechanical noxious stimuli but are responsive to thermal noxious stimuli. Two such units were in fact encountered and are described below.

\section{Topography of nociresponsive and non-nociresponsive receptive ficlds}

Receptive field locations and sizes were documented for all units studied. Within VPL, the WDR type nociresponsive cells seemed randomly scattered between LT type units. The receptive field locations and sizes of these WDR cells were similar to, and varied correspondingly with, neighboring LT cells (see Fig. 8). Since, as shown above, the mean ongoing rate was also the same for WDR and LT cells in VPL, the only way to distinguish between the two groups was by systematic testing of all these units by noxious stimulation. In Figure 8 , two WDR units are interspersed between LT units in VPL. The location and size of the receptive fields of these WDR units are similar to, and continuous with, the surrounding LT units' receptive fields. The unit at the border between VPL and VPI is an LT type cell with a small receptive field at the tip of digit 2 . The next three more
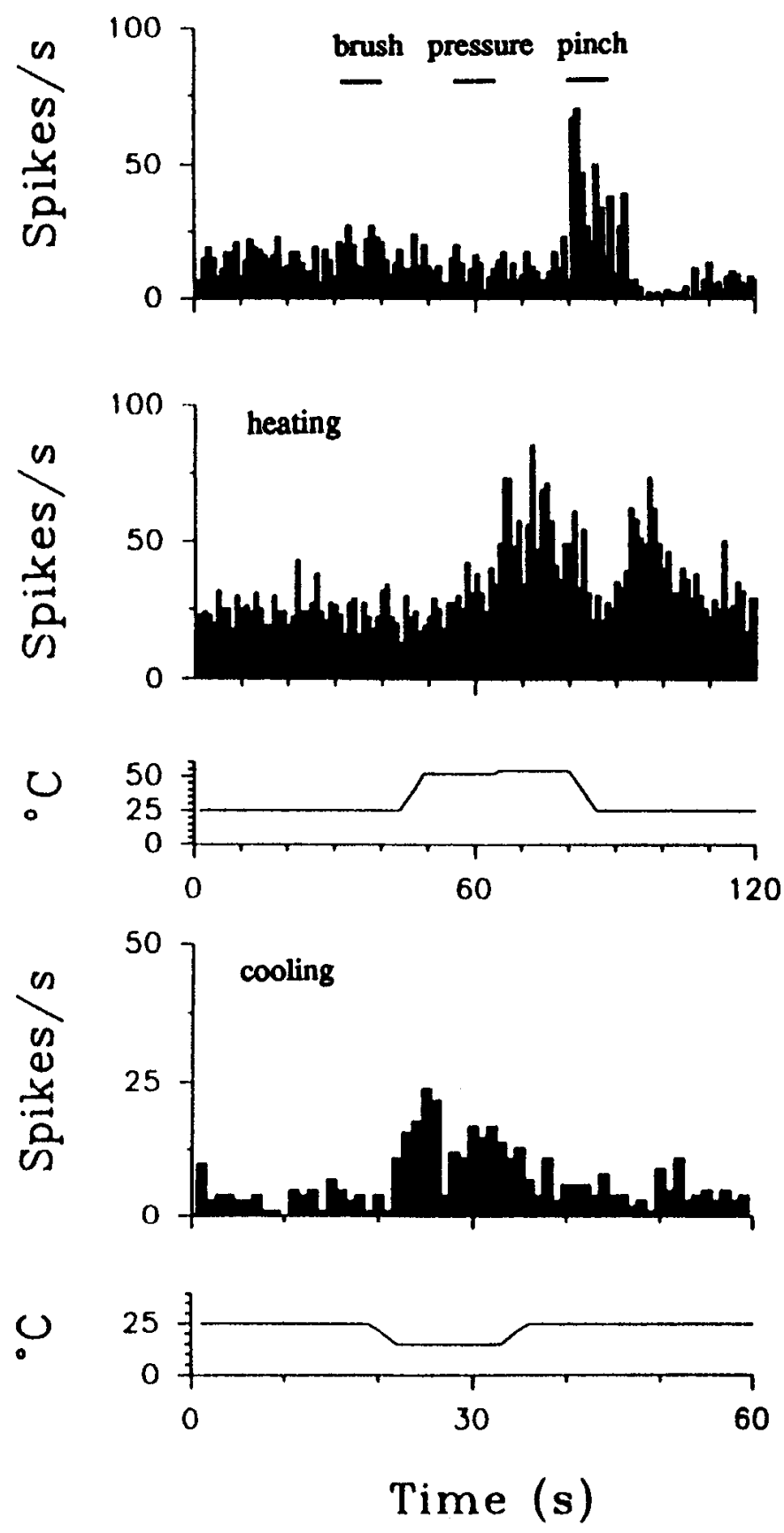

Figure 5. Response of a cell to mechanical and thermal noxious and innocuous cooling. This nociceptive-specific type unit was found in VPI; its rcceptive field covered the hand and forearm. With mechanical stimulation the unit activity increased only during noxious pinching $(10 \mathrm{sec}$ duration; top graph). Heating the receptive field from a baseline of $25^{\circ} \mathrm{C}$ to $52^{\circ} \mathrm{C}$ did not change the ongoing activity. However, increasing the temperature by another $2^{\circ} \mathrm{C}\left(54^{\circ} \mathrm{C}\right.$ ) resulted in increased activity (middle graph). Cooling the receptive field from $25^{\circ} \mathrm{C}$ baseline to $15^{\circ} \mathrm{C}$ resulted in increased activity (bottom graph). The time scale in the bottom graph is different from the other two graphs.

ventral units are nociceptive and their receptive field sizes increase from the digits (unit 11), to lower arm (unit 12), eventually to encompass the hand and the lips and the ear (unit 13; this receptive field is discontinuous but not all sites in between were tested). In Figure 9, all nociresponsive cells were in VPI and were WDR type. Similar to the other track, as the electrode moves more ventrally in VPI the receptive field sizes tend to 

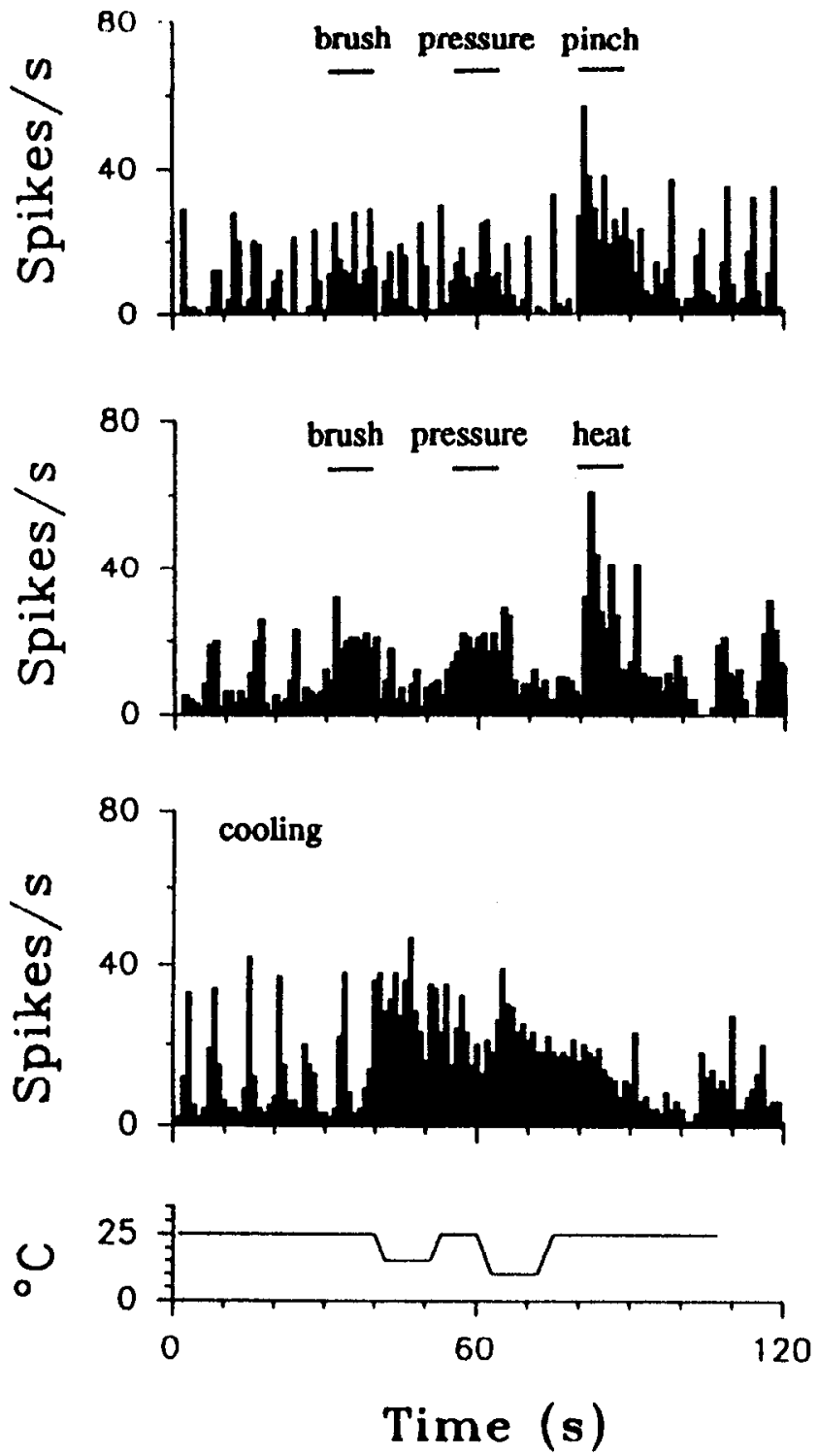

Figure 6. Response of a wide-dynamic-range type cell to mechanical and thermal noxious stimuli and to cooling. This unit was found in VPI; its receptive field covered the hand and forearm. With mechanical stimulation the unit activity increased during brushing and further increased during noxious pinching (top graph). Heating the receptive field with a probe (about $55^{\circ} \mathrm{C}$ ) increased the unit activity above that of brushing or pressure (middle graph). Cooling the receptive field from $25^{\circ} \mathrm{C}$ baseline to $15^{\circ} \mathrm{C}$ and then to $5^{\circ} \mathrm{C}$ resulted in increased activity (bottom graph).

become larger. Both tracks (Figs. 8,9) as well as other tracks show that the incidence of nociresponsive cells increases ventrally, as the electrode passes into VPI.

Three recording tracks were found traversing through the anterior part of PO. One of the tracks with nine recording sites is shown in Figure 10. The receptive field sequence in this track starts at the shoulder, goes to the foot, then jumps back to the axilla and goes down to the leg again, suggesting a dual body map. Four of the nine units were nociresponsive, and three of these four nociresponsive units were WDR type. The last unit (unit 9) responded to brushing on the leg and pressure but not to pinch; however, noxious heating of the receptive field increased the ongoing activity. One other unit with similar re- sponses to noxious heating but unresponsive to mechanical noxious stimuli was found in VPI; it was NS type, and responded only to noxious heat or cold.

For the WDR type neurons the receptive fields determined by brushing or touching the skin were usually comparable in size and location to the receptive fields determined by noxious stimulation. However, there were some striking exceptions to this rule. In four units (two in VPL and two in VPI) the lowthreshold receptive fields, although overlapping, were two to three times larger than the high-threshold receptive fields. For instance, in a WDR unit, located in VPL, the low-threshold receptive field spanned from the chest to the whole arm and hand, while the high-threshold receptive field was limited to the chest. In two units (one in VPI and one in VPM) the lowthreshold receptive fields were smaller than the high-threshold receptive fields. For example, a WDR type unit in VPI had responses to brushing the dorsal hairs of digits 1 and 2 and to hcating and cooling thesc digits of the hand; when tested with pinch the unit also responded throughout the dorsal portion of the arm.

\section{Somatotopy of VPI neurons}

Thirteen electrode tracts, in six of seven animals, passed through VPI. The locations and receptive fields of 48 VPI neurons were determined. Figure 11 shows the locations of all these units in VPI (non-nociceptive and nociceptive) and the body part where their receptive fields were located. Mediolaterally, the receptive fields follow the same organization as that in VP. Namely, the receptive fields are located on the face medially, on the hand and arm more laterally, and on the leg and foot even more laterally. The receptive fields of VPL cells just dorsal to the tracks in VPI are also shown in Figure 11. Comparing these receptive fields to the tracks in VPI, 6 of the 13 tracks show that as the electrode moves into VPI the receptive fields jump from one body part to another. At least in the medial tracks, larger receptive fields seem located more ventrally. Similar to the organization of nociceptive neurons in VP, the receptive field sizes and location of VPI nociresponsive cells are in continuity with the non-nociresponsive cells in the same tracks.

\section{Receptive fields of nociresponsive cells}

The locations and sizes of receptive fields responding to noxious stimulation are shown in Figure $12 A-C$, for all the nociresponsive cells studied. Eleven of the 17 VPL nociresponsive cells had receptive fields located on the hand or arm, primarily due to the search strategy, and two units had receptive fields on the neck and face (Fig. 12A); the two VPM nociresponsive cells had receptive fields on the face. In contrast, the receptive fields of nociresponsive cells in VPI had a much larger face representation, 8 of 23 (Fig. 12B). These VPI cells with face inputs were usually the most ventral units encountered in the recording tracks and tended to be located more medial than VPI cells with forelimb receptive fields. Also, eight (of 23) of the receptive field sizes of the nociresponsive cells in VPI were larger than the ones found in VPL, while 13 of these cells had receptive fields similar in size to those in VPL. A statistical analysis of the receptive fields' locations, comparing between VP and VPI for the number of units with receptive fields including or excluding the face, results in borderline significance $\left(\chi^{2}\right.$ test, $\left.p=0.06\right)$. The overall number of nociresponsive cells in $\mathrm{PO}$ was small. However, their receptive field locations seem similar to those in VPI, but their receptive field sizes seem larger than those in VPI (Fig. 12C). 


\section{A LT in VPL and VPI}
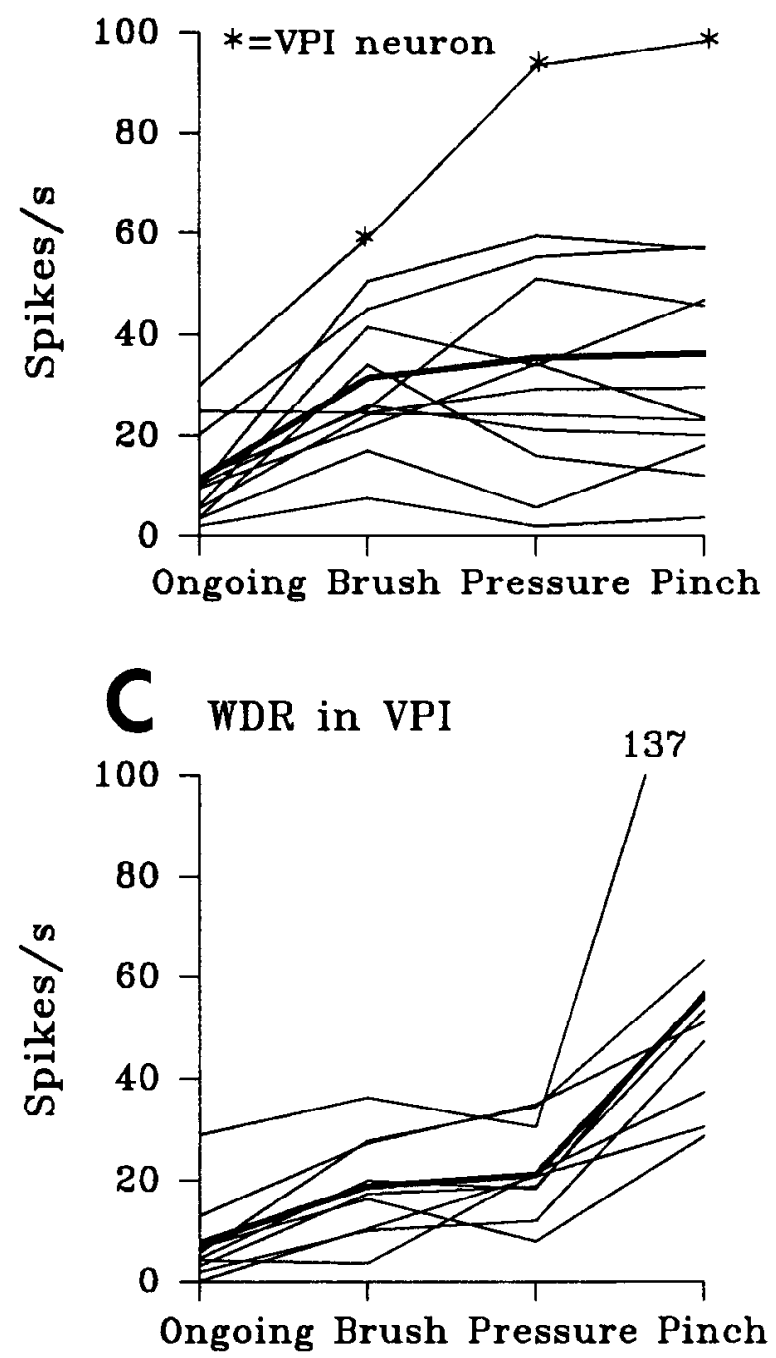

B

WDR in VPL

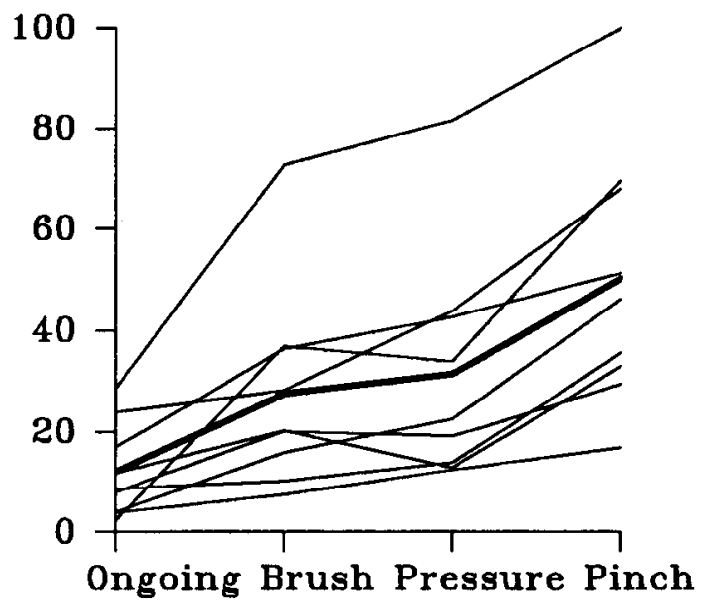

D

HT in VPI and PO

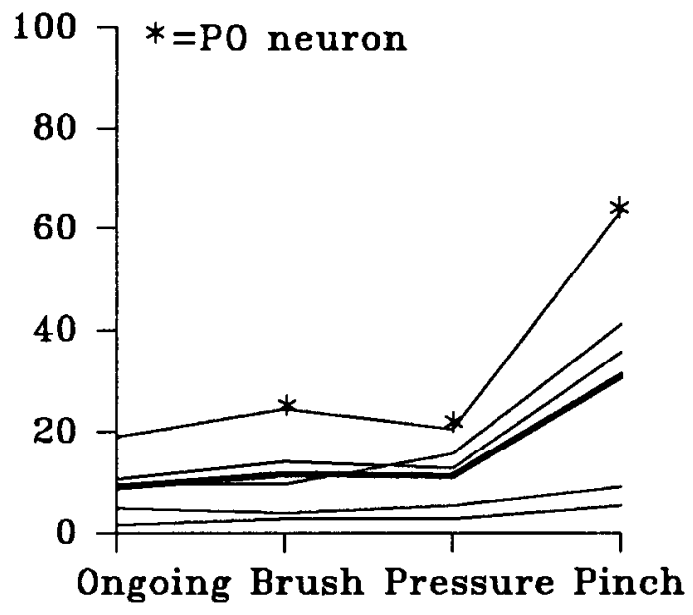

Figure 7. Individual and population stimulus response properties of lateral thalamic cells to mechanical stimulation. $A$, Responses of 12 units classified as non-nociceptive. Some units increase their responses during pressure, others decrease. The responses to pinch are always less than or equal to the responses during brush or pressure. The population response is increased during brushing and pressure (thick line). $B$, Responses of nine units classified as wide-dynamic-range type and located in VPL. Some units increase their responses during pressure; others decrease. The responses to pinch are always more than the responses during brush or pressure. The population responses increase more during pinching than during brushing and pressure (thick line). $C$, Responses of 10 units classified as wide-dynamic-range type and located in VPI. Some units increase their responses during pressure; others decrease. The population responses are similar to those in $B$. $D$, Responses of five units (four were located in VPI, one was in PO indicated by asterisks) classified as high threshold. The population response is increased only during pinch (thick line).

The location and size of receptive fields of nociceptive neurons were relatively stable over the time span of monitoring (around 1-2 hr). However, in three cases the receptive fields dramatically enlarged. The receptive field of an NS type unit, located in VPI, expanded from one digit (digit 3) to include the two neighboring digits as well (digits 2,3 , and 4), after a few repeated pinch stimuli. The innocuous and noxious receptive field of a WDR unit, located in VPL, was initially limited to a small area of the radial side of the forearm. This receptive field responded to innocuous and noxious. stimuli and was constant for this test duration (about $30 \mathrm{~min}$ ). This receptive field expanded, over a time span of $30 \mathrm{~min}$, during which the animal did not receive any somatic test stimuli, to include the radial side of the whole arm and digits 3,4 , and 5 . Increased responses could then be elicited to both brushing and pinching from this enlarged region. Another WDR unit, in VPL, initially exhibited responses to touch of the dorsal hand and wrist and to pinching on the same receptive field. Subsequently (after repeated testing with noxious stimulation over a couple of minutes), the lowthreshold response expanded to include the whole arm and hand.

\section{Location of nociresponsive cells relative to spinothalamic terminals}

The electrode tracks in Figures 8 and 9 show the locations of the units relative to the spinothalamic track terminals. Some of the spinothalamic terminals are in close apposition to the recording sites. This relationship was quantified by determining the number of recording sites that were within (overlapping), or outsidc (nonoverlapping), $100 \mu \mathrm{m}$ of spinothalamic terminals. Since the spinothalamic terminals were labeled by injecting the cervical enlargement, this relationship was determined only for units with receptive fields that at least partially included prox- 

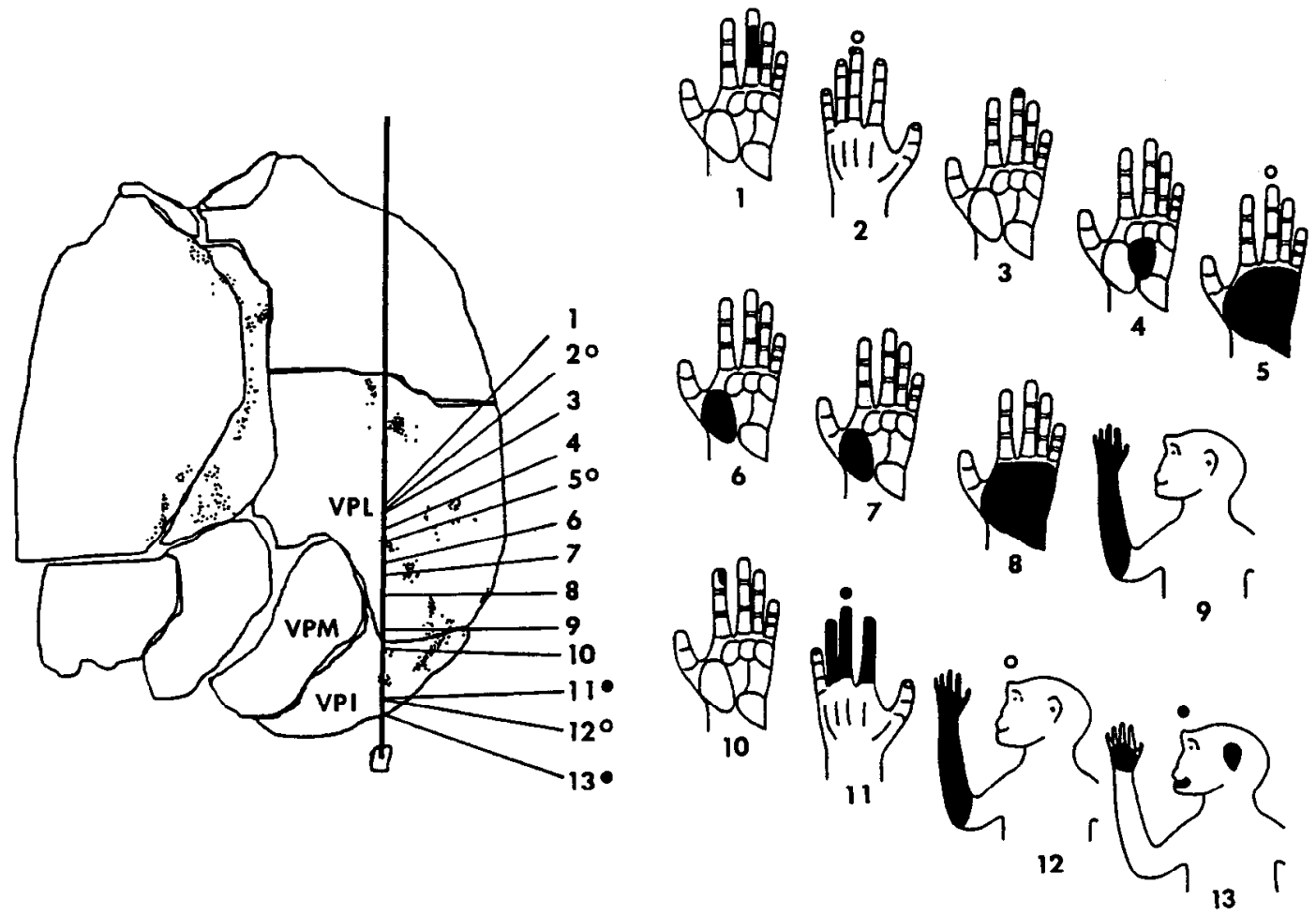

Figure 8. Units isolated in a single electrode track, their receptive fields, and their locations relative to the spinothalamic terminations. In this track 13 units were isolated. Three of those (units 2, 5, and 12) were wide-dynamic-range type (open circles), two (units 11 and 13) were nociceptivespecific type (solid circles), and the remainder were non-nociceptive. Spinothalamic terminals are shown with black dots. The receptive fields are shown in black.

Figure 9. Units isolated in another electrode track. In this track 14 units were isolated. Four of those (units 11 , 12,13 , and 14) were wide-dynamicrange type (open circles). Spinothalamic terminals are shown with black dots. The receptive fields are shown in black.

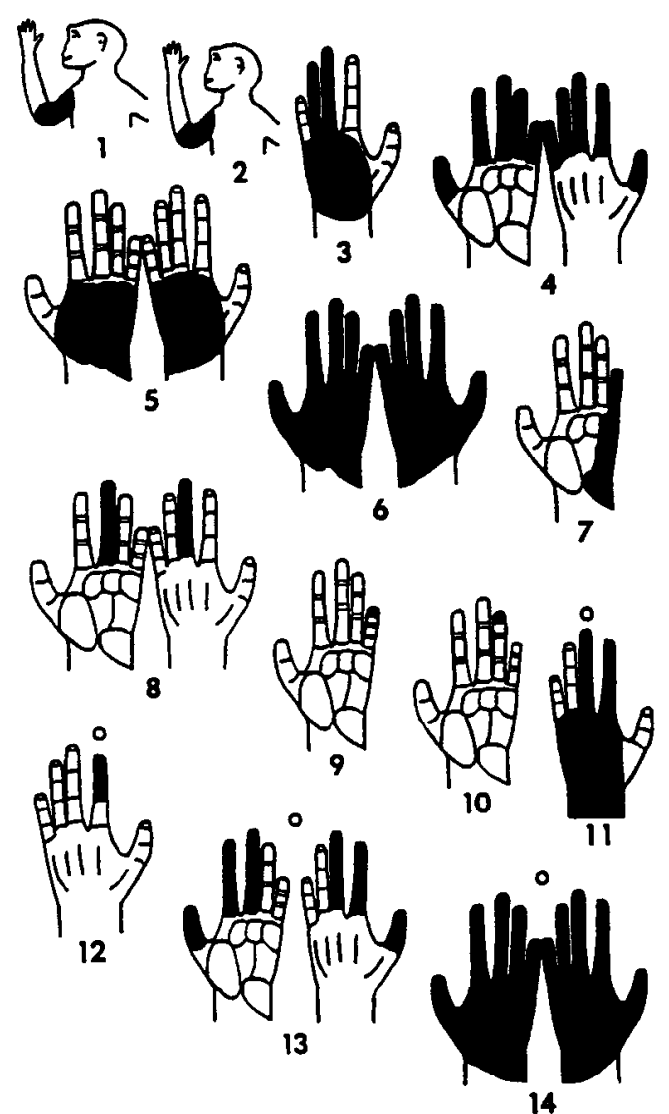



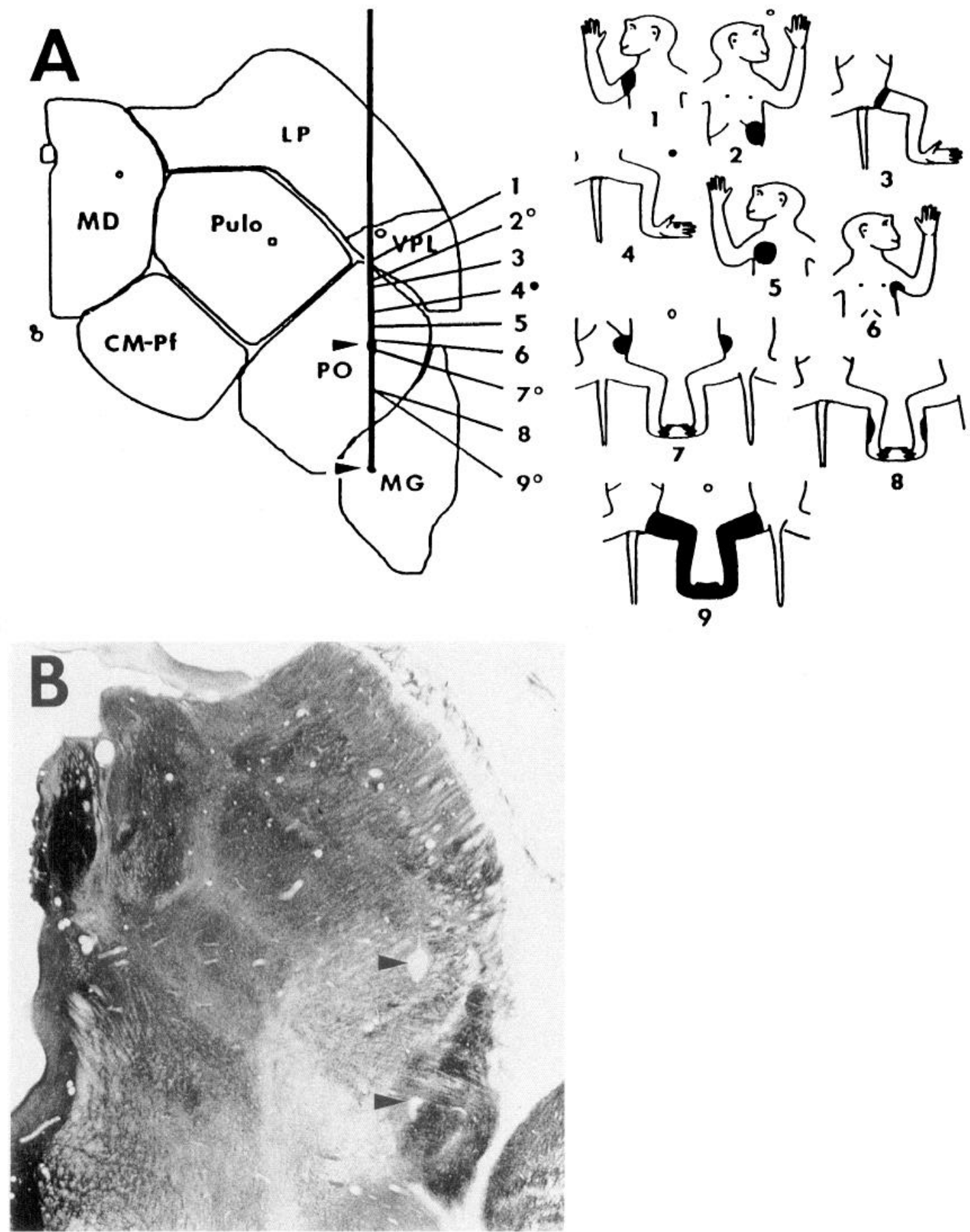

Figure 10. A, Units isolated in an electrode track passing through PO. In this track nine units were isolated. Three of those (units 2, 7, and 9) were wide-dynamic-range type (open circles); unit 4 was NS type (solid circle). Spinothalamic terminals were present in the PO at this level (not illustrated since they were not analyzed). The receptive fields are shown in black. In $B$ the corresponding cytochrome oxidase-stained section is shown. There are two electrolytic lesions (arrows), one marking the location of unit 7, the other the end of the track.

imal forelimb dermatomes. Table 2 summarizes this relation for neurons located in VPL and VPI. Only $20-26 \%$ of the LT neurons, in VPL and VPI, were classified as overlapping, and the rest as nonoverlapping. Nociresponsive cells in VPL had a similar distribution; only three of nine units were classified as overlapping. On the other hand the locations of nociresponsive units in VPI were more closely correlated with spinothalamic terminals; 8 of 11 were classified as overlapping. A $2 \times 2$ con- tingency table of the VPI LT and non-LT cells, with and without spinothalamic overlap, resulted in a statistically significant difference $\left(\chi^{2}\right.$ test, $\left.p<0.05\right)$.

\section{Discussion}

These results indicate that in the squirrel monkey, (1) neurons in VPL, VPM, VPI, and PO respond to noxious somatic stimuli. (2) There is a specific organization for the nociresponsive cells 


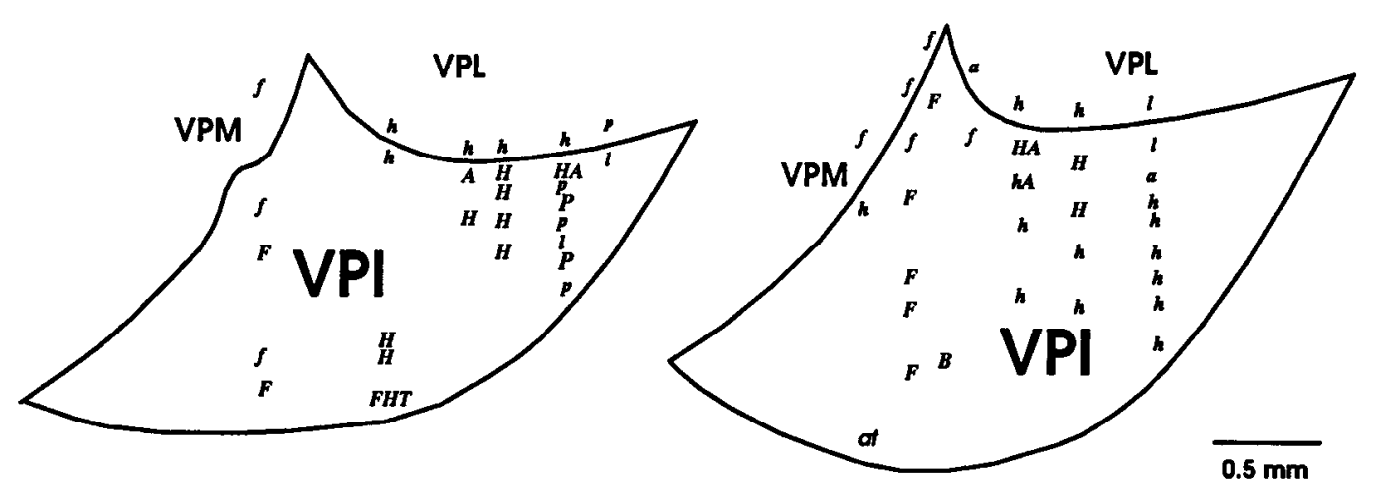

Figure 11. Somatic representation in VPI. All tracks passing through VPI and all neurons with receptive fields in these tracks are illustrated. The body part where the units' receptive fields were located are shown at the recording sites. The receptive fields of these neurons were on the body side contralateral to the thalamic recordings. Receptive fields located on the face are indicated as $f, F$; on the hand as $h, H$; on the arm as $a, A$; on the trunk as $t, T$; on the leg as $l$; and on the foot as $p, P$; on half of the body including both limbs as $B$. Receptive fields shown in $u p p e r c a s e$ indicate nociceptive inputs from these body parts. Receptive fields shown in lowercase indicate that only non-nociceptive stimuli could activate the neurons.

in these regions. The VP nociceptive neurons have WDR type responses, while VPI and PO neurons have both WDR and NS type response properties. The incidence of nociresponsive cells is much higher in VPI, and perhaps in PO, as compared to VP. A subpopulation of the cells responsive to noxious pinch also respond to noxious thermal stimuli. An even smaller group of these cells is also responsive to innocuous cooling. The receptive field locations and sizes for VPL nociresponsive cells are indistinguishable from the nonnociceptive cells in the same region. The body map represented in VPI mediolaterally seems similar to that in VPL; however, more ventral in VPI the receptive fields tend to be more complex and discontinuous, and can include hand and face. Also, the receptive field sizes of VPI cells tend to be larger than those for VPL cells located just dorsally. (3) The relations between spinothalamic terminals and the locations of the nociresponsive units are different for VPL and VPI. Within VPL, the probability of receiving spinothalamic inputs seems to be the same irrespective of the response properties of the units. On the other hand, in VPI, nociresponsive units have a higher probability of receiving spinothalamic inputs than non-nociresponsive units.

\section{Comparison with other thalamic physiologic studies}

The overall somatotopic organization of VP agrees with earlier studies (Kaas et al., 1984; Chung et al., 1986). However, the receptive field sizes of $\mathrm{LT}$ and nociresponsive neurons in VP

\begin{tabular}{|c|c|c|c|c|c|c|c|}
\hline & \multicolumn{3}{|c|}{ VPL } & \multicolumn{3}{|c|}{ VPI } & \multirow{2}{*}{$\begin{array}{l}\text { Total } \\
+ \\
\end{array}$} \\
\hline & - & + & $\%$ & - & + & $\%$ & \\
\hline Non-noc. & 75 & 27 & 26 & 8 & 2 & 20 & $26 \%$ \\
\hline WDR & 5 & 3 & \multirow{2}{*}{33} & 1 & 7 & 73 & \multirow{3}{*}{$55 \%$} \\
\hline NS & 1 & 0 & & 2 & 1 & 15 & \\
\hline Total & 81 & 30 & 27 & 11 & 10 & 48 & \\
\hline
\end{tabular}

Cells that were found at a distance greater than $100 \mu \mathrm{m}$ from spinothalamic terminals $(-)$ are assumed to be unable to receive spinothalamic inputs. Cells that are within $100 \mu \mathrm{m}$ from spinothalamic terminals $(+)$ putatively receive spinothalamic inputs (i.e., overlapping). $\%$ is percent $+/(+$ and - ) in each category. seem larger and their body locations seem more disjoint in comparison to Kaas et al.'s study. This difference is likely due to the different recording strategies used; Kaas et al. continuously mapped multiunit responses while we only studied wellisolated individual neurons.

The proportion of nociresponsive cells encountered in the thalamus seems to vary with the species used, for example, rat (Guilbaud et al., 1980), cat (Kniffki and Craig, 1985; Martin et al., 1990), and monkey (Chung et al., 1986); search stimulus employed; and the region of the thalamus examined (Dong et al., 1978; Chung et al., 1986; Brüggemann et al., 1993, in press). Thus, generalizations on the subject are hard. However, the present and our earlier studies (Martin et al., 1990) indicate that the incidence of nociresponsive neurons in the lateral thalamus is much higher in squirrel monkeys than in cats.

Somatic responsive cells have been reported in the monkey VPI (Pollin and Albe-Fessard, 1979; Dykes et al., 1981), but this region has not been systematically studied in relation to nociception. Dykes et al. (1981) have implied that cells in this area are primarily responsive to Pacinian type inputs. However, we could not identify thalamic cells with Pacinian type inputs. This observation is in agreement with studies conducted in the cat in Kniffki's laboratory, where units with Pacinian type inputs have not been observed in and around VPL or VPM in the lateral thalamus (Kniffki and Vahle-Hinz, 1987; Vahle-Hinz et al., 1987; Vahle-Hinz and Kniffki, in press). The high incidence of nociresponsive cells, especially NS type cells, within the monkey VPI is similar to results obtained by Kniffki and VahleHinz in VPMvp of the cat (Kniffki and Vahle-Hinz, 1987; VahleHinz and Kniffki, in press).

Casey and Morrow (1987, 1992) have recorded from nociresponsive cells from the VPL in squirrel monkeys. These experiments were conducted in awake behaving monkeys. Surprisingly, the incidence of nociceptive cells (13-18\%), their locations within VPL and VPI, and their response types, very closely agree with our results. Similar to the present study, they find that most nociceptive cells in VPL are of WDR type and many (15 of 29 units, Casey and Morrow, 1987; about 20 of 23, Morrow and Casey, 1992) are scattered within the core of the nucleus. Therefore, the anesthesia used in our study does not seem to alter significantly the response properties of the 

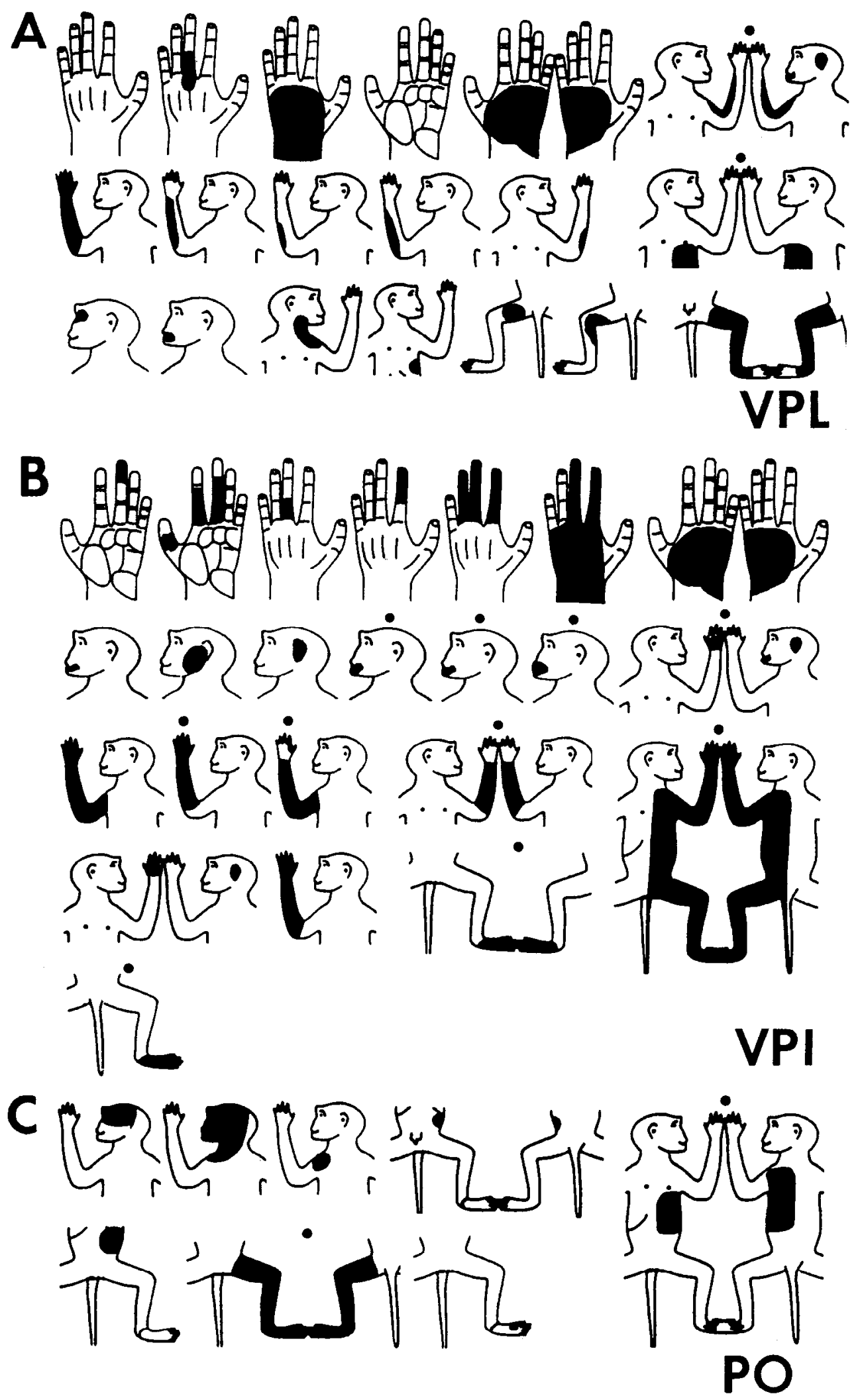

Figure 12. The receptive field locations, sizes, and response types for all nociresponsive cells found in VPL and $\operatorname{VPM}(A)$, VPI $(B)$, and PO $(C)$. Nociceptive-specific type cells are indicated by solid circles. All other cells are widedynamic-range type.

nociresponsive cells, at least in VPL. Nociresponsive cells in the macaque are also scattered within the core of VPL and the majority are WDR type (Chung et al., 1986). However, using an electrical search stimulus, Chung et al. report a higher incidence of nociresponsive cells $(50 \%)$ and a significant number of NS type cells (14\%) in the macaque VPL. Nociresponsive and thermoceptive cells were recently described in VPM of the awake behaving macaque (Bushnell et al., 1993). These units, WDR type and NS type, were found concentrated along the borders of VPM. On the other hand, the responses of these VPM cells to thermal stimuli and the continuity of their receptive fields with surrounding innocuous cells are reminiscent of the VPL 
nociresponsive neurons. Both the raccoon (Simone et al., 1993) and the rat (Guilbaud et al., 1980) seem more similar to the primate in that nociresponsive cells are found within the core of VPL. However, the incidence of nociresponsive cells in areas equivalent to VPI remain unknown in the macaque, rat, and raccoon.

\section{Cooling responsive cells}

This is the first report of units with cooling responses in VPL, VPI, and PO. Bushnell et al. (1993) reported on 12 cooling responsive cells in VPM in the awake macaque monkey. Seven of these cells were WDR type and had heating responses as well. The remainder were low-threshold mechanical-responsive cells. We did not test the LT type cells with cooling stimuli. The response properties shown by Bushnell et al. for the nociceptive cooling responsive cells are very similar to the cells we have found in VPL, VPI, and PO. Recently it was reported that microstimulation in the human lateral thalamus may evoke "cool" and "warm" perceptions (Lenz et al., 1993a). Lenz et al. (1993b) have now been able to record from threc cooling rcsponsive cells in the lateral thalamus of the awake human. These units had greater responses to noxious heat and had phasic responses to cooling. These cells were all located in an area that closely corresponds to VPI and perhaps anterior PO in the squirrel monkey (see below).

\section{Nociresponsive cells in $P O$}

The only physiologic study of nociresponsive cells in the monkey PO was done by Perl and Whitlock (1961), who could not find any nociceptive cells in PO. A recent study in the rat (Diamond et al., 1992) shows that POm has a complete somatotopy and the cells with inputs from the vibrissae can be activated from multiple hairs. Krubitzer and Kaas (1992) have proposed that the cat POm is equivalent to the monkey anterior pulvinar, while Jones (1985) has proposed that the cat POm is equivalent to the anteriormost portion of monkey PO. Therefore, the correspondence between the rat, cat, and monkey associative somatosensory regions remains unsettled. In the present study a very limited portion (the most anterior part) of the monkey PO was explored. However, the neurons in this region had relatively small, contralateral receptive fields and many had nociceptive responses. Most physiologic studies of $\mathrm{PO}$ have shown large bilateral receptive fields (Poggio and Mountcastle, 1960; Perl and Whitlock, 1961; Curry, 1972; Berkley, 1973). This difference is probably due to the specific portion of $\mathrm{PO}$ studied here. Overall, our knowledge of the role of this region in nociception remains to be further evaluated.

\section{Spinothalamic inputs to VPL and VPI nociresponsive cells}

Our results show that the primate VPI is involved in processing nociceptive-specific information (largest incidence of NS type cells) and that this information is transmitted through the spinothalamic terminals in the area. Spinal cord lamina I neurons have the largest concentration of NS type cells in the spinal cord (for review and references, see Willis, 1985) and makeup about one-third of all spinothalamic cells (Apkarian and Hodge, 1989a; Stevens et al., 1989). These observations together suggest that VPI receives nociceptive information primarily from spinal cord lamina I neurons, through the dorsal spinothalamic pathway (Apkarian and Hodge, 1989b). The primate VPL has primarily WDR type nociceptive neurons. This implies that its spinothalamic inputs are from spinal cord lamina V-VIII cells, and not from lamina I cells, through the ventral spinothalamic pathway (Apkarian and Hodge, 1989b). It is also possible that some of the WDR type cells in VPL are due to convergent inputs from NS type and LT typc projections from the spinal cord and dorsal column nuclei. The lack of correspondence between response types and STT contacts in VPL may be partially due to technical difficulties of matching recording sites with STT terminals. This, however, seems unlikely since there was a statistically significant correspondence between response types and STT locations for VPI cells. The more likely and more interesting implication is that the STT is not the primary source of nociceptive input to VPL and that other pathways may be important in nociceptive information transmission to VPL nociceptive cells.

\section{Cortical targets}

The cells examined in this study were not tested for cortical targets. Therefore, we have no idea as to the relative number of the cells that might be cortically projecting. However, we assume that the large majority, especially those located in VPL, were neurons with large somata and hence were projecting cells since their responses could be recorded over many hours. Many of the cells in VPI were only recorded for shorter periods. This is consistent with the cells in VPI being smaller in size than those in VPL. However, we assume that many of the VPI cells studied were also cortically projecting.

In an earlier study we estimated, using anatomic techniques, the maximum putative spinothalamic inputs to SI-projecting cells in the thalamus (Gingold et al., 1991). The proportions of SI-projecting cells that can receive spinothalamic inputs (i.e., overlapping) at the soma, proximal dendrites, and distal dendrites were counted. These proportions were $8 \%, 24 \%$, and $42 \%$, respectively. In the present study we estimate that $18.5 \%$ of the neurons in VPL, VPI, and PO are nociresponsive. Therefore, assuming that nociceptive information is relayed to the thalamus mainly through the spinothalamic tract, it can be concluded that this input is directed mainly to the proximal dendrites, a conclusion demonstrated anatomically by others (Ralston et al., 1985). Moreover, the overlap study showed that the proportion of SI projecting cells with spinothalamic inputs in VPI was twice as large as the proportion of the same population cells in VPL (Gingold et al., 1991). The present physiologic study shows that the likelihood of encountering nociresponsive cells increases four times when the electrode is in VPI as compared to VPL. Therefore, both studies agree that VPI has a higher proportion of nociresponsive cells, although the relative likelihoods do not match. In another recent anatomic study we estimated the maximum putative spinothalamic inputs to SII projecting cells in the thalamus (Stevens et al., 1993). The study showed that most of these cells are located in the ventral portion of VPL and in VPI and PO, with equal probabilities across the three nuclei (around 30\%). It is quitc likcly, therefore, that a significant proportion of the nociresponsive cells encountered in the present study had projections to SII.

Our anatomic work (Gingold et al., 1991; Stevens et al., 1993) as well as many other earlier studies (for review and references, see Kaas and Pons 1988; Krubitzer and Kaas, 1992) show that primary and secondary somatosensory cortices (SI and SII) receive their main inputs from VPL and VPI, respectively. Since VPL nociresponsive cells are mostly WDR type, we conclude that SI nociresponsive cells should be mainly of the WDR type, which is consistent with physiologic studies of SI (Kenshalo and 
Isensee, 1983; Kenshalo et al., 1988). Also, since VPI cells exhibit high incidence of NS type responses, we conclude that SII should show similar prevalence of NS type cells. This conclusion agrees with work of Dong et al. (1989, and personal communication) showing that more than $50 \%$ of the nociresponsive cells in the somatosensory regions of SII and area $7 \mathrm{~b}$ in the awake monkey are NS type. The correspondence between response types of nociceptive cells in SI and VPL, and in SII and VPI, implies that processing of nociceptive information at these cortical areas is distinct from that of innocuous information processing. Innocuous information is known to occur through serial channels from VPL to SI and then to SII (Pons et al., 1992). We propose that nociceptive information flow is primarily in parallel, with distinct types of information going from VPL to SI, and from VPI to SII.

\section{Implications for human pain perception}

Hassler (1970) has described that stimulating a region called V.c.pc. in humans (Jones, 1990, agrees with Hassler that V.c.pc. corresponds to VPI in the monkey) results in a well-localized contralateral sensation of pain. This observation was the initial inspiration that led Kniffki and colleagues (Kniffki and Mizumura, 1983; Kniffki and Craig, 1985) to study the response properties of cells in the ventral border of the cat VP. These studies were the first convincing demonstration for the existence of nociresponsive neurons around VP in mammals in a region that closely approximates Hassler's initial report.

More recently, recordings and microstimulation were performed in the lateral thalamus of chronic pain or motor disorder patients (Lenz et al., 1987, 1988, 1993a,b; Tasker et al., 1987; Dostrovsky et al., 1991). These studies indicate the existence of a lateral thalamic region, just ventral and posterior to the main somatosensory nucleus, the stimulation of which results in localized contralateral thermal or pain reports in unanesthetized subjects, and single-unit recordings show a small number of cells with activity correlated with noxious stimulation. Dostrovsky et al. (1991) state "the unpleasant sensations were evoked in most cases by stimulation at sites at or close to (within 0.5 $\mathrm{mm}$ above to $3 \mathrm{~mm}$ below) the ventral border of VP. The evoked sensations were described as painful in about half of these cases...."We measured the dorsoventral extent of the human VPI (V.c.pc. of Hassler) from cresyl violet-/and myelin-stained tissue (prepared by Watkins, donated to us by R. B. King). The largest dorsoventral extent of the human VPI is $2-3 \mathrm{~mm}$. Therefore, VPI seems to be consistent, in both size and location, with the lateral thalamic region, stimulation of which evokes pain perception in humans. Moreover, there seems to be a close correspondence between the large increase in frequency of evoking pain perception by stimulating this area in humans and the frequency with which we encounter nociresponsive cells in VPI. Recently Lenz et al. (1993b) have recorded from 10 (of 185) nociresponsive cells in the lateral thalamus of humans. One of these neurons was in V.c (monkey VP) and was WDR type. The others were all NS thermoreceptive type and were located inferior and posterior to V.c, an area again closely corresponding to monkey VPI, and perhaps including anterior PO. The authors point out that the nociresponsive neurons were more frequently recorded at sites where electrical stimulation evoked pain sensations. Therefore, we propose that VPI in the cat, monkey, and human is a unique region in the lateral thalamus that participates in the conscious perception of the discriminative properties of nociceptive inputs. This raises the question regarding the role of the WDR type nociresponsive cells within VP. It is possible that these cells participate in the conscious pain perception as well, but that stimulation within VP does not cause pain perception because these cells have a more sparse spatial distribution than the nociresponsive cells in VPI. Alternately, the nociresponsive cells within VP may play a modulatory role to adjust the level of excitability of lemniscal inputs as a function of nociceptive inputs (for evidence, see Stea and Apkarian, 1992; Apkarian et al., in press).

\section{Conclusion}

There are at least two distinct somatotopically arranged nociceptive maps in the lateral thalamus, located in VPL and VPI; the somatotopy of PO nociceptive cells remains to be studied more extensively. These two systems have different inputs, response properties, and output targets in the cortex. The response properties of these neurons fit the criteria necessary for participating in the discriminative aspects of pain perception (Price and Dubner, 1977), by detecting stimulus location, duration, and intensity with a local representational code. The following companion article (Brüggeman et al., 1994) demonstrates the response properties of cells in the same lateral thalamic region when the units are tested with visceral stimuli.

\section{Appendix}

Abbreviations

CL central lateral nucleus

CM-Pf centromedian-parafascicular nuclei

LD lateral dorsal nucleus

LP posterior lateral nucleus

MD mediodorsal nucleus

MG medial geniculate nucleus

Pf parafascicular nucleus

PO postcrior complcx

POm medial division of posterior neucleus

Pulo oral or anterior part of pulvinar

VP ventral posterior complex

VPI ventral posterior inferior nucleus

VPL ventral posterior lateral nucleus

VPM ventral posterior medial nucleus

R thalamic reticular nucleus

ZI zona incerta

\section{References}

Albe-Fessard D, Berkley KJ, Kruger L, Ralston HJ III, Willis WD Jr (1985) Diencephalic mechanisms of pain sensation. Brain Res Rev 9:217-296.

Apkarian AV, Hodge CJ (1989a) The primate spinothalamic pathways. I. A quantitative study of the cells of origin of the spinothalamic pathway. J Comp Neurol 288:447-473.

Apkarian AV, Hodge CJ (1989b) The primate spinothalamic pathways. II. The cells of origin of the dorsolateral and ventral spinothalamic pathways. J Comp Neurol 288:474-492.

Apkarian AV, Stea RA, Bolanowski SJ (in press) Suppression of the sense of touch by pain: a touch gate. Somatosens Motor Res, in press.

Berkley KJ (1973) Response properties of cells in ventrobasal and posterior group nuclei of the cat. J Neurophysiol 36:940-952.

Besson J-M, Chaouch A (1987) Peripheral and spinal mechanisms of nociception. Physiol Rev 67:67-186.

Brüggemann J, Vahle-Hinz C, Kniffki K-D (1993) Representation of the urinary bladder in the lateral thalamus of the cat. $\mathrm{J}$ Neurophysiol $17: 482-491$.

Brüggemann J, Shi T, Apkarian AV (1994) Squirrel monkey lateral thalamus. II. Viscerosomatic convergent representation of urinary bladder, colon, and esophagus. J Neurosci 14:6796-6814.

Brüggemann J, Vahle-Hinz C, Apkarian AV, Kniffki K-D (in press) Somato-visceral convergence in thalamic regions of the cat. J Neurophysiol, in press. 
Bushnell MC, Duncan GH, Termblay N (1993) Thalamic VPM nucleus in the behaving monkey. I. Multimodal and discriminative properties of thermosensitive neurons. J Neurophysiol 69:739-752.

Casey KL (1966) Unit analysis of nociceptive mechanisms in the thalamus of the awake squirrel monkey. J Physiol (Lond) 29:727750.

Casey KL, Morrow TJ (1983) Ventral posterior thalamic neurons differentially responsive to noxious stimulation of the awake monkey. Science 221:675-677.

Casey KL, Morrow TJ (1987) Nociceptive neurons in the ventral posterior thalamus of the awake squirrel monkey: observations on identification, modulation, and drug effects. In: Thalamus and pain (Besson J-M, Guilbaud D, Peschanski M, eds), pp 211-226. Amsterdam: Elsevier.

Chung JM, Lee KH, Surmeier DJ, Sorkin LS, Kim J, Willis WD (1986) Response characteristics of neurons in the ventral posterior lateral nucleus of the monkey thalamus. J Neurophysiol 56:370-390.

Craig AD, Burton H (1981) Spinal and medullary lamina I projection to nucleus submedius in medial thalamus: a possible pain center. $\mathrm{J}$ Neurophysiol 45:443-466.

Craig AD, Kniffki K-D (1985) The multiple representation of nociception in the spinothalamic projection of lamina I cells in the cat. In: Development, organization, and processing in somatosensory pathways (Rowe M, Willis WD, eds), pp 347-353. New York: Liss.

Curry MJ (1972) The exteroceptive properties of neurones in the somatic part of the posterior group (PO). Brain Res 44:439-462.

Diamond ME, Armstrong-James M, Ebner FF (1992) Somatic sensory responses in the rostral sector of the posterior group (POm) and in the ventral posterior medial nucleus (VPM) of the rat thalamus. $J$ Comp Neurol 318:462-476.

Dong WK, Ryu H, Wagman IH (1978) Nociceptive responses of neurons in medial thalamus and their relationship to spinothalamic pathways. J Neurophysiol 41:1592-1613.

Dong WK, Salonen LD, Kawakam Y, Shiwaku T, Kaukoranta EM, Martin RF (1989) Nociceptive responses of trigeminal neurons in SII-7b cortex of awake monkeys. Brain Res 484:314-324.

Dostrovsky JO, Wells FEB, Tasker RR (1991) Pain sensations evoked by stimulation in human thalamus. In: Processing and inhibition of nociceptive information (Inoki $\mathrm{Y}$, Shigenaga $\mathrm{Y}$, Tohyama $\mathrm{M}$, eds), pp 115-120. Amsterdam: Elsevier.

Dykes RW, Sur M, Merzenich MM, Kaas JH, Nelson RJ (1981) Regional segregation of neurons responding to quickly adapting, slowly adapting, deep and Pacinian receptors within thalamic ventroposterior lateral and ventroposterior inferior nuclei in the squirrel monkey (Saimiri sciureus). Neuroscience 6:1687-1692.

Gingold SI, Greenspan JD, Apkarian AV (1991) Anatomic evidence of nociceptive inputs to primary somatosensory cortex: relationship between spinothalamic terminals and thalamocortical cells in squirrel monkeys. J Comp Neurol 308:467-490.

Guilbaud G, Peschanski M, Gautron M, Binder D (1980) Neurones responding to noxious stimulation in VB complex and caudal adjacent regions in the thalamus of the rat. Pain 8:303-318.

Hassler R (1970) Supranuclear structures-dichotomy of facial pain conduction in the diencephalon. In: Trigeminal neuralgia-pathogenesis and pathophysiology (Hassler R, Walker AE, eds), pp 123138. Philadelphia: Saunders.

Head H, Holmes G (1911) Sensory disturbances from cerebral lesions. Brain 34:102-254.

Hodge CJ Jr, Apkarian AV (1990) The spinothalamic track. Crit Rev Neurobiol 5:363-397.

Honda CN, Mense S, Perl ER (1983) Neurons in ventrobasal region of cat thalamus selectively responsive to noxious mechanical stimulation. J Neurophysiol 49:662-673.

Jones EG (1985) The thalamus. New York: Plenum.

Jones EG (1990) Correlation and revised nomenclature of ventral nuclei in the thalamus of human and monkey. Stereotact Funct Neurosurg 54:1-20.

Kaas JH, Pons TP (1988) The somatosensory system of primates. Comp Prim Biol 4:421-468.

Kaas JH, Neison RJ, Dykes RW, Merzenich MM (1984) The somatotopic organization of the ventroposterior thalamus of the squirrel monkey, Saimiri sciureus. J Comp Neurol 266:111-140.

Kenshalo DR Jr, Isensee O (1983) Responses of primate SI cortical neurons to noxious stimuli. J Neurophysiol 50:1479-1496.

Kenshalo DR Jr, Giesler GJ Jr, Leonard RB, Willis WD (1980) Responses of neurons in primate ventral posterior lateral nucleus to noxious stimuli. J Neurophysiol 43:1594-1614.
Kenshalo DR Jr, Chudler EH, Anton F, Dubner R (1988) SI nociceptive neurons participate in the encoding process by which monkeys perceive the intensity of noxious thermal stimulation. Brain Res 454: 378-382.

Kniffki K-D, Craig AD (1985) The distribution of nociceptive neurons in the cat's lateral thalamus: the dorsal and ventral periphery of VPL. In: Development, organization, and processing in somatosensory pathways (Rowe M, Willis WD Jr, eds), pp 375-382. New York: Liss

Kniffki K-D, Mizumura K .(1983) Responses of neurons in VPL and VPL-VL region of the cat to algesic stimulation of muscle tendon. J Neurophysiol 49:649-661.

Kniffki K-D, Vahle-Hinz C (1987) The periphery of the cat's ventroposteromedial nucleus (VPMp): nociceptive neurones. In: Thalamus and pain (Besson J-M, Guilbaud G, Peschanski M, eds), pp 245-257. Amsterdam: Elsevier.

Krubitzer LA, Kaas JH (1992) The somatosensory thalamus of monkeys: cortical connections and a redefinition of nuclei in marmosets. J Comp Neurol 319:123-140.

Lenz FA, Tasker RR, Dostrovsky JO, Kwan HC, Gorecki J, Hirayama T, Murphy JT (1987) Abnormal single-unit activity recorded in the somatosensory thalamus of a quadriplegic patient with central pain. Pain 31:225-236.

Lenz FA, Dostrovsky JO, Tasker RR, Yamashiro K, Kwan HC, Murphy JT (1988) Single-unit analysis of the human ventral thalamic nuclear group: somatosensory responses. J Neurophysiol 59:299-316.

Lenz FA, Seike M, Richardson RT, Lin YC, Baker FH, Khoja I, Jaeger CI, Gracely RH (1993a) Thermal and pain sensations evoked by microstimulation in the area of human ventrocaudal nucleus. J Neurophysiol 70:200-212.

Lenz FA, Scikc M, Lin YC, Baker FII, Rowland LH, Gracely RH, Richardson RT (1993b) Neurons in the area of human thalamic nucleus ventralis caudalis $(\mathrm{Vc})$ respond to painful heat stimuli. Brain Res 623:235-240.

Martin RJ, Apkarian AV, Hodge CJ Jr (1990) Ventrolateral and dorsolateral ascending spinal cord pathway influence on thalamic nociception in cat. J Neurophysiol 64:1400-1412.

Melzack R, Casey KL (1968) Sensory, motivational, and central control determinants of pain. In: The skin senses (Kenshalo DR, ed), pp 423-443. Springfield, IL: Thomas.

Mesulam M-M (1978) Tetramethyl benzidine for horseradish peroxidase neurohistochemistry: a non-carcinogenic blue reaction product with superior sensitivity for visualizing neural afferents and efferents. J Histochem Cytochem 26:106-117.

Morrow TJ, Casey KL (1992) State-related modulation of thalamic somatosensory responses in the awake monkey. J Neurophysiol 67: 305-317.

Perl ER, Whitlock DG (1961) Somatic stimuli exciting spinothalamic projections to thalamic neurons in cat and monkey. Exp Neurol 3:256296.

Poggio GF, Mountcastle VB (1960) A study of the functional contributions of the lemniscal and spinothalamic systems to somatic sensitivity. Bull Johns Hopkins Hosp 106:266-316.

Pollin B, Albe-Fessard D (1979) Organization of somatic thalamus in monkeys with and without section of dorsal spinal tracts. Brain Res 173:431-449.

Pons TP, Garraghty PE, Mishkin M (1992) Serial and parallel processing of tactual information in somatosensory cortex of rhesus monkeys. J Neurophysiol 68:518-527.

Price DD, Dubner R (1977) Neurons that subserve the sensory-discriminative aspects of pain. Pain 3:307-338.

Ralston HJ, Peschanski M, Ralston DD (1985) Fine structure of spinothalamic track axons and terminals in rat, cat, and monkey demonstrated by the orthograde transport of lectin conjugated to horseradish peroxidase. In: Advances in pain research and therapy, Vol 9 (Fields HL, Dubner R, Cervero F, eds), pp 269-275. New York: Raven.

Rosene DL, Roy NJ, Davis BJ (1986) A cryoprotection method that facilitates cutting frozen sections of whole monkey brains for histological and histochemical processing without freezing artifact. J Histochem Cytochem 34:1301-1315.

Schmued LC (1990) A rapid, sensitive histochemical stain for myelin in frozen brain sections. J Histochem Cytochem 38:717-720.

Shi T, Stevens RT, Tessier J, Apkarian AV (1993) Spinothalamocortical inputs nonpreferentially innervate the superficial deep cortical layers of SI. Neurosci Lett 160:209-213.

Simone DA, Hanson ME, Bernau NA, Pubols BH Jr (1993) Nocicep- 
tive neurons of the raccoon lateral thalamus. J Neurophysiol 69:318328.

Smith MV, Apkarian AV, Hodge CJ Jr (1991) Somatosensory response properties of contralaterally projecting spinothalamic and nonspinothalamic neurons in the second cervical segment of the cat. J Neurophysiol 66:83-102.

Stea RA, Apkarian AV (1992) Pain and somatosensory activation. Trends Neurosci 15:250-251.

Stevens RT, Hodge CJ Jr, Apkarian AV (1989) Medial, intralaminar, and lateral terminations of lumbar spinothalamic tract neurons: a fluorescent double-label study. Somatosens Motor Res 6:285-308.

Stevens RT, London SM, Apkarian AV (1993) Spinothalamocortical projections to the secondary somatosensory cortex (SII) in squirrel monkey. Brain Res 631:241-246.

Tago H, Kimura H, Maeda T (1986) Visualization of detailed acetylcholinesterase fiber and neuron staining in rat brain by a sensitive histochemical procedure. J Histochem Cytochem 34:1431-1438.

Tasker RR, Gorecki J, Lenz FA, Hirayama T, Dostrovsky JO (1987)
Thalamic microelectrode recording and microstimulation in central and deafferentation pain. Appl Neurophysiol 50:414-417.

Vahle-Hinz C, Kniffki K-D (in press) Somatic nociceptive neurons in the periphery of the cat's thalamic ventral posteromedial nucleus (VPMp). J Neurophysiol, in press.

Vahle-Hinz C, Freund I, Kniffki K-D (1987) Nociceptive neurons in the ventral periphery of the cat thalamic ventroposteromedial nucleus. In: Fine afferent nerve fibers and pain (Schmidt RF, Schaible $H-G$, Vahle-Hinz C, eds), pp 439-450. Weinheim: VCH.

Willis WD Jr (1985) The pain system: the neural basis of nociceptive transmission in the mammalian nervous system. New York: Karger.

Wong-Riley M (1979) Changes in the visual system of monocularly sutured or enucleated cats demonstrable with cytochrome oxidase histochemistry. Brain Res 171:11-28.

Yokota T, Asato F, Koyama N, Masuda T, Taguchi H (1988) Nociceptive body represcntation in nucleus ventralis posterolateralis of cat thalamus. J Neurophysiol 60:1714-1727. 